\title{
Hypercomplex Algebraic Geometry
}

\author{
Dominic Joyce \\ published as Quart. J. Math. Oxford 49 (1998), 129-162.
}

\section{Introduction}

It is well-known that sums and products of holomorphic functions are holomorphic, and the holomorphic functions on a complex manifold form a commutative algebra over $\mathbb{C}$. The study of complex manifolds using algebras of holomorphic functions upon them is called complex algebraic geometry.

The purpose of this paper is to develop an analogue of complex algebraic geometry, in which the complex numbers $\mathbb{C}$ are replaced by the quaternions $\mathbb{H}$. The natural quaternionic analogue of a complex manifold is called a hypercomplex manifold. A class of $\mathbb{H}$-valued functions on hypercomplex manifolds will be defined, called q-holomorphic functions, that are analogues of holomorphic functions on complex manifolds. Now, the set of holomorphic functions on a complex manifold is a commutative algebra over $\mathbb{C}$. Therefore one asks: does the set of q-holomorphic functions on a hypercomplex manifold have an analogous algebraic structure, and if so, what is it?

We shall show that the q-holomorphic functions on a (noncompact) hypercomplex manifold do indeed possess a rich algebraic structure. To describe it, we shall introduce a theory of quaternionic algebra, which is a quaternionic analogue of real linear algebra. This theory is built on three building blocks: AH-modules, the analogues of vector spaces, AH-morphisms, the analogues of linear maps, and the quaternionic tensor product, the analogue of tensor product of real vector spaces.

As far as the author can tell, these ideas seem to be new. They enable us to construct algebraic structures over $\mathbb{H}$ as though $\mathbb{H}$ were a commutative field. Quaternionic algebra describes the algebraic structure of hypercomplex manifolds in a remarkable way, and it seems to be the natural language of hypercomplex algebraic geometry, the algebraic geometry of hypercomplex manifolds.

We believe that quaternionic algebra is worth studying for its own sake. It has many similarities with linear algebra over $\mathbb{R}$ or $\mathbb{C}$, which is why the analogies between complex and quaternionic theories work so well, but there are also deep differences, which give quaternionic algebra a flavour all of its own.

Quillen [12] has given a sheaf-theoretic interpretation of the ideas of quaternionic algebra, based on a previous version of this paper. He finds a contravariant equivalence between a class of $\mathrm{AH}-$-modules and regular sheaves on a real form of $\mathbb{C P}^{1}$. Regular sheaves on $\mathbb{C P}^{1}$ are equivalent to representations of the Kronecker quiver, and out of such a representation Quillen constructs an AHI-module. Under Quillen's equivalence stable AHI-modules correspond to regular vector bundles over the real form of $\mathbb{C P}^{1}$. 
We start in $\S 2$ by reviewing the quaternions and defining the concepts of AHI-modules and AHI-morphisms. Section 3 defines hypercomplex manifolds and q-holomorphic functions, and shows that the q-holomorphic functions on a hypercomplex manifold are an AHI-module. The key to the algebraic side of this paper is the definition in $\S 4$ of the quaternionic tensor product, and an exploration of some of its properties.

Section 5 defines $\mathrm{H}$-algebras, the quaternionic analogues of commutative algebras, and shows that the q-holomorphic functions on a hypercomplex manifold form an H-algebra. We briefly discuss the problem of recovering a hypercomplex manifold from its H-algebra, which leads to the idea of an algebraic geometry of hypercomplex manifolds. In $\S 6$ hyperkähler manifolds are defined, and we explain how the H-algebra of a hyperkähler manifold acquires an additional algebraic structure, making it into an HP-algebra.

Sections 7-9 study the quaternionic algebra of finite-dimensional AHI-modules in greater depth. In $\S 7$ a series of examples are given which illustrate differences between real and quaternionic algebra. Sections 8 and 9 concern two special classes of AHI-modules, stable and semistable AHI-modules. Amongst other things, we show that the quaternionic tensor product $U \otimes_{\mathbb{H}} V$ of two stable AHI-modules is stable, and give a formula for $\operatorname{dim} U \otimes_{\mathbb{H}} V$. By restricting to stable AHI-modules, some of the differences between real and quaternionic algebra are resolved, and the analogy between real and quaternionic algebra becomes more complete.

Finally, $§ \S 10-12$ give geometrical applications of the theory to hypercomplex and hyperkähler manifolds. Section 10 studies q-holomorphic functions on $\mathbb{H}$, and $\S \S 11$ and 12 prove some new results on hyperkähler manifolds with large symmetry groups, including an algebraic construction of Kronheimer's hyperkähler metrics on coadjoint orbits [10], [11].

Acknowledgements. I would like to thank John Cernes, Peter Kronheimer, Peter Neumann, and Simon Salamon for interesting conversations.

\section{AHI-modules}

The quaternions $\mathbb{H}$ are $\mathbb{H}=\left\{r_{0}+r_{1} i_{1}+r_{2} i_{2}+r_{3} i_{3}: r_{0}, \ldots, r_{3} \in \mathbb{R}\right\}$, and quaternion multiplication is given by

$$
i_{1} i_{2}=-i_{2} i_{1}=i_{3}, \quad i_{2} i_{3}=-i_{3} i_{2}=i_{1}, \quad i_{3} i_{1}=-i_{1} i_{3}=i_{2}, \quad i_{1}^{2}=i_{2}^{2}=i_{3}^{2}=-1 .
$$

The quaternions are an associative, noncommutative algebra. If $q=r_{0}+r_{1} i_{1}+r_{2} i_{2}+r_{3} i_{3}$ then we define the conjugate $\bar{q}$ of $q$ by $\bar{q}=r_{0}-r_{1} i_{1}-r_{2} i_{2}-r_{3} i_{3}$. Then $\overline{(p q)}=\bar{q} \bar{p}$ for $p, q \in \mathbb{H}$. The imaginary quaternions are $\mathbb{I}=\left\langle i_{1}, i_{2}, i_{3}\right\rangle$.

Definition 2.1 The following notation will be used throughout the paper. If $V$ is a vector space, then $V^{*}$ is the dual space and id $: V \rightarrow V$ the identity map. Also, $\operatorname{dim} V$ means the dimension of $V$ as a real vector space, even if $V$ is an $\mathbb{H}$-module. A (left) $\mathbb{H}$-module is a real vector space $U$ with an action of $\mathbb{H}$ on the left. We write this action $(q, u) \mapsto q \cdot u$ or $q u$, for $q \in \mathbb{H}$ and $u \in U$. In this paper, all $\mathbb{H}$-modules will be left $\mathbb{H}$-modules. Let $U$ be an $\mathbb{H}$-module. We define the dual $\mathbb{H}$-module $U^{\times}$to be the vector space of linear maps $\alpha: U \rightarrow \mathbb{H}$ that satisfy $\alpha(q u)=q \alpha(u)$ for all $q \in \mathbb{H}$ and $u \in U$. If $q \in \mathbb{H}$ and $\alpha \in U^{\times}$, define $q \cdot \alpha$ by $(q \cdot \alpha)(u)=\alpha(u) \bar{q}$ for $u \in U$. Then $q \cdot \alpha \in U^{\times}$, and $U^{\times}$is a (left) $\mathbb{H}$-module. Dual $\mathbb{H}$-modules behave just like dual vector spaces. 
Now we define AHI-modules, which should be thought of as the quaternionic analogues of real vector spaces.

Definition 2.2 Let $U$ be an $\mathbb{H}$-module. Let $U^{\prime}$ be a real vector subspace of $U$, that need not be closed under the $\mathbb{H}$-action. Define a real vector subspace $U^{\dagger}$ of $U^{\times}$by

$$
U^{\dagger}=\left\{\alpha \in U^{\times}: \alpha(u) \in \mathbb{I} \text { for all } u \in U^{\prime}\right\} .
$$

Conversely, $U^{\dagger}$ determines $U^{\prime}$, at least for finite-dimensional $U$, by

$$
U^{\prime}=\left\{u \in U: \alpha(u) \in \mathbb{I} \text { for all } \alpha \in U^{\dagger}\right\}
$$

We define an augmented $\mathbb{H}$-module, or AHI-module, to be a pair $\left(U, U^{\prime}\right)$, such that if $u \in U$ and $\alpha(u)=0$ for all $\alpha \in U^{\dagger}$, then $u=0$. Usually we will refer to $U$ as an AHI-module, implicitly

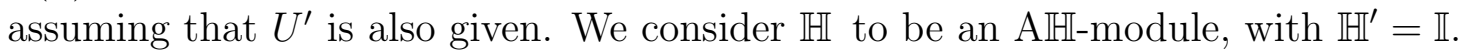

The condition that $u=0$ if $\alpha(u)=0$ for all $\alpha \in U^{\dagger}$ is important. Its purpose should become clear in $\S \S 3$ and 4 . We can interpret $U^{\times}$as the dual of $U$ as a real vector space, and then $U^{\dagger}$ is the annihilator of $U^{\prime}$. Thus if $U$ is finite-dimensional, $\operatorname{dim} U^{\prime}+\operatorname{dim} U^{\dagger}=\operatorname{dim} U=\operatorname{dim} U^{\times}$. Here are the natural concepts of linear map between AHI-modules, and submodules of AHI-modules.

Definition 2.3 Let $U, V$ be AHI-modules. Let $\phi: U \rightarrow V$ be a linear map satisfying $\phi(q u)=q \phi(u)$ for each $q \in \mathbb{H}$ and $u \in U$. Such a map is called $\mathbb{H}$-linear. We say that $\phi$ is an AHI-morphism, if $\phi: U \rightarrow V$ is $\mathbb{H}$-linear and $\phi\left(U^{\prime}\right) \subset V^{\prime}$. If $\phi$ is an isomorphism of $\mathbb{H}$-modules and $\phi\left(U^{\prime}\right)=V^{\prime}$, we say $\phi$ is an AHI-isomorphism. If $\phi: U \rightarrow V$ and $\psi: V \rightarrow W$ are AHI-morphisms, then $\psi \circ \phi: U \rightarrow W$ is an AHI-morphism.

Let $U, V$ be AHI-modules and $\phi: U \rightarrow V$ an AHI-morphism. Define an $\mathbb{H}$-linear map $\phi^{\times}: V^{\times} \rightarrow U^{\times}$by $\phi^{\times}(\beta)(u)=\beta(\phi(u))$ for $\beta \in V^{\times}$and $u \in U$. Then $\phi\left(U^{\prime}\right) \subset V^{\prime}$ implies

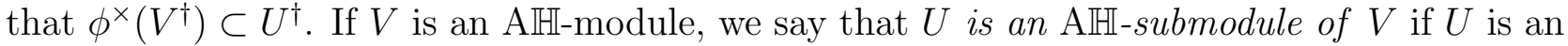
$\mathbb{H}$-submodule of $V$ and $U^{\prime}=U \cap V^{\prime}$. As $U^{\dagger}$ is the restriction of $V^{\dagger}$ to $U$, if $\alpha(u)=0$ for all $\alpha \in U^{\dagger}$ then $u=0$, so $U$ is an AHI-module.

\section{Hypercomplex manifolds and q-holomorphic functions}

Let $M$ be a manifold of dimension $2 n$. A complex structure $I$ on $M$ is a smooth tensor $I_{a}^{b}$ on $M$ that satisfies the equations

$$
I_{a}^{b} I_{b}^{c}=-\delta_{a}^{c} \quad \text { and } \quad I_{c}^{d} \nabla_{b} I_{a}^{c}-I_{c}^{d} \nabla_{a} I_{b}^{c}+I_{a}^{c} \nabla_{c} I_{b}^{d}-I_{b}^{d} \nabla_{c} I_{a}^{d}=0,
$$

where $\nabla$ is any torsion-free connection on $T M$. A manifold $M$ with a complex structure $I$ is called a complex manifold. Let $M$ be a complex manifold and $f: M \rightarrow \mathbb{C}$ a differentiable function. Write $f=f_{0}+i f_{1}$, where $f_{0}, f_{1}: M \rightarrow \mathbb{R}$. Then $f$ is called holomorphic if

$$
d f_{0}+I\left(d f_{1}\right)=0
$$

on $M$, where (3.2) is called the Cauchy-Riemann equation.

Here are the quaternionic analogues of complex manifolds and holomorphic functions. Let $M$ be a manifold of dimension $4 n$. A hypercomplex structure on $M$ is a triple $\left(I_{1}, I_{2}, I_{3}\right)$ on 
$M$, where $I_{j}$ is a complex structure on $M$, and $I_{1} I_{2}=I_{3}$. In index notation this condition is written $\left(I_{1}\right)_{a}^{b}\left(I_{2}\right)_{b}^{c}=\left(I_{3}\right)_{a}^{c}$. If $M$ has a hypercomplex structure, it is called a hypercomplex manifold. Since $I_{1}, I_{2}, I_{3}$ satisfy the quaternion relations (2.1), each tangent space $T_{m} M$ is an $\mathbb{H}$-module isomorphic to $\mathbb{H}^{n}$. For more information about hypercomplex manifolds, see for instance [13, p. 137-9], [7] and [8].

Now let $M$ be a hypercomplex manifold, and $f: M \rightarrow \mathbb{H}$ a smooth function. Then $f=f_{0}+f_{1} i_{1}+f_{2} i_{2}+f_{3} i_{3}$, where $f_{0}, \ldots, f_{3}$ are smooth real functions. Define a 1 -form $D(f)$ on $M$ by

$$
D(f)=d f_{0}+I_{1}\left(d f_{1}\right)+I_{2}\left(d f_{2}\right)+I_{3}\left(d f_{3}\right) .
$$

We define a q-holomorphic function on $M$ to be a smooth function $f: M \rightarrow \mathbb{H}$ for which $D(f)=0$. Equation (3.3) is the natural quaternionic analogue of the Cauchy-Riemann equation (3.2).

Q-holomorphic functions on $\mathbb{H}$ were studied, a long time ago, by Fueter and his collaborators. In 1935, Fueter defined q-holomorphic functions on $\mathbb{H}$, which he called 'regular functions', and went on to develop the theory of quaternionic analysis, by analogy with complex analysis. This theory included analogues of Cauchy's Theorem, Cauchy's integral formula, the Laurent expansion, and so on. However, as far as the author knows, Fueter and his school did not discover the quaternionic tensor product or the theory of multiplying q-holomorphic functions that will be explained in this paper. Accounts of the theory, with references, are given by Sudbery [14] and Deavours [4].

Next we will show that the q-holomorphic functions on a hypercomplex manifold form an $\mathrm{A} \mathbb{H}$-module, in the sense of Definition 2.2.

Definition 3.1 Let $M$ be a hypercomplex manifold. Define $A_{M}$ be the real vector space of q-holomorphic functions on $M$. Let $f \in A_{M}$ and $q \in \mathbb{H}$, and define a function $q \cdot f$ on $M$ by $(q \cdot f)(m)=q(f(m))$ for all $m \in M$. By equation $(3.3), D(q \cdot f)=0$ if $D(f)=0$, so that $q \cdot f \in A_{M}$. This gives an action of $\mathbb{H}$ on $A_{M}$, which makes $A_{M}$ into an $\mathbb{H}$-module.

Now define a real vector subspace $A_{M}^{\prime}$ in $A_{M}$ by

$$
A_{M}^{\prime}=\{f \in A: f(m) \in \mathbb{I} \text { for all } m \in M\} .
$$

For each $m \in M$, define $\theta_{m}: A_{M} \rightarrow \mathbb{H}$ by $\theta_{m}(f)=f(m)$. Then $\theta_{m} \in A_{M}^{\times}$, and if $f \in A_{M}^{\prime}$ then $\theta_{m}(f) \in \mathbb{I}$, so that $\theta_{m} \in A_{M}^{\dagger}$. Suppose $f \in A_{M}$, and $\alpha(f)=0$ for all $\alpha \in A_{M}^{\dagger}$. Since $\theta_{m} \in A_{M}^{\dagger}$, $f(m)=0$ for each $m \in M$, and so $f=0$. Thus we have proved that the vector space $A_{M}$ of q-holomorphic functions on $M$ is an AHI-module.

If $M$ is a hypercomplex manifold, then it has three complex structures $I_{1}, I_{2}, I_{3}$. But these are not the only complex structures. If $a_{1}, a_{2}, a_{3} \in \mathbb{R}$ with $a_{1}^{2}+a_{2}^{2}+a_{3}^{2}=1$, then $a_{1} I_{1}+a_{2} I_{2}+a_{3} I_{3}$ is also a complex structure on $M$, so that there is a family of complex structures on $M$ parametrized by the 2 -sphere $\mathcal{S}^{2}$, which should be regarded as the unit sphere in $\mathbb{I}$.

Let $\Sigma_{j=1}^{3} a_{j}^{2}=1$, so that $I=\Sigma_{j} a_{j} I_{j}$ is a complex structure on $M$, and let $i=\Sigma_{j} a_{j} i_{j} \in \mathbb{I}$. Then $i^{2}=-1 \in \mathbb{H}$, and $\langle 1, i\rangle$ is a subalgebra of $\mathbb{H}$ isomorphic to $\mathbb{C}$. Let $f_{0}, f_{1}$ be real functions on $M$, and suppose that $f_{0}+f_{1} i$ is a holomorphic function on $M$ with respect to $I$. Then $d f_{0}+I\left(d f_{1}\right)=0$ on $M$ by (3.2). Substituting $I=\Sigma_{j} a_{j} I_{j}$, we see that

$$
D\left(f_{0}+a_{1} f_{1} i_{1}+a_{2} f_{1} i_{2}+a_{3} f_{1} i_{3}\right)=d f_{0}+I_{1}\left(a_{1} d f_{1}\right)+I_{2}\left(a_{2} d f_{2}\right)+I_{3}\left(a_{3} d f_{3}\right)=0,
$$

and from (3.3) we see that $f_{0}+f_{1} i$ is a q-holomorphic function on $M$. 
Thus, any $\mathbb{C}$-valued function on $M$ that is holomorphic with respect to one of the $\mathcal{S}^{2}$ family of complex structures, can also be regarded as a q-holomorphic $\mathbb{H}$-valued function, by embedding $\mathbb{C}$ in $\mathbb{H}$ in the appropriate way. This tells us two important things. Firstly, on small open sets in any complex manifold there are many holomorphic functions. Therefore, on small open sets of a hypercomplex manifold, there are many q-holomorphic functions. If $\operatorname{dim} M>4$, the equation (3.3) is overdetermined, so one would expect few solutions or none, but this is not the case.

Secondly, the product of two holomorphic functions is holomorphic. Therefore, it is possible in some circumstances to take two q-holomorphic functions on a hypercomplex manifold, multiply them together, and get a third q-holomorphic function. So, we expect some sort of multiplicative structure on $A_{M}$, the AHI-module of q-holomorphic functions on $M$. However, in general the product of two q-holomorphic functions is not q-holomorphic. Thus, $A_{M}$ is not an algebra in the simple, obvious sense. We shall explain in the next two sections how to describe the multiplicative structure on $A_{M}$.

Now we define some special AIII-modules $X_{q}$.

Definition 3.2 Let $q \in \mathbb{I}$ be nonzero. Define an AHI-module $X_{q}$ by $X_{q}=\mathbb{H}$, and $X_{q}^{\prime}=\{p \in$ $\mathbb{H}: p q=-q p\}$. It is easy to show that $X_{q}^{\prime} \subset \mathbb{I}$ and $\operatorname{dim} X_{q}^{\prime}=\operatorname{dim} X_{q}^{\dagger}=2$. For example, if $q=i_{1}$, then $X_{i_{1}}^{\prime}=\left\langle i_{2}, i_{3}\right\rangle \subset \mathbb{H}$.

We can use these AHI-modules to characterize the holomorphic functions in the set of q-holomorphic functions.

Lemma 3.3 Let $M$ be a hypercomplex manifold. Let $i \in \mathbb{I}$ satisfy $i^{2}=-1$, and let $I$ be the corresponding complex structure on $M$. Suppose that $f=f_{0}+i f_{1}$ is a holomorphic function on $M$ w.r.t. I. Define a map $\phi_{f}: X_{i} \rightarrow A_{M}$ by $\phi_{f}(q)=q \cdot f$. Then $\phi_{f}$ is an AHI-morphism. Conversely, if $\phi: X_{i} \rightarrow A_{M}$ is an AH-morphism, then $f=\phi(1) \in A_{M}$ takes values in $\langle 1, i\rangle \subset \mathbb{H}$, and is holomorphic w.r.t. $\mathbb{I}$.

Proof. Suppose for simplicity that $i=i_{1}$ and $I=I_{1}$. The proof for general $i \in \mathbb{I}$ works the same way. As $f$ is holomorphic w.r.t. $I_{1}, f \in A_{M}$ from above, and if $f \in A_{M}$ then $q \cdot f \in A_{M}$. Thus $\phi_{f}$ maps $X_{i_{1}} \rightarrow A_{M}$, and is clearly $\mathbb{H}$-linear. We must prove that $\phi_{f}\left(X_{i_{1}}^{\prime}\right) \subset A_{M}^{\prime}$. As $X_{i_{1}}^{\prime}=\left\langle i_{2}, i_{3}\right\rangle$, it is enough to show that $\phi_{f}\left(i_{2}\right)$ and $\phi_{f}\left(i_{3}\right)$ lie in $A_{M}^{\prime}$. Now $\phi_{f}\left(i_{2}\right)=f_{0} i_{2}-f_{1} i_{3}$, where $f_{0}$ and $f_{1}$ are real functions. Thus, $\phi_{f}\left(i_{2}\right)$ takes values in $\mathbb{I}$ and so $\phi_{f}\left(i_{2}\right) \in A_{M}^{\prime}$ by (3.4). Similarly, $\phi_{f}\left(i_{3}\right)=f_{1} i_{2}+f_{0} i_{3}$, so $\phi_{f}\left(i_{3}\right) \in A_{M}^{\prime}$. Therefore $\phi_{f}$ is an AHI-morphism.

For the next part, let $\phi(1)=f_{0}+f_{1} i_{1}+f_{2} i_{2}+f_{3} i_{3}$. Since $X_{i_{1}}^{\prime}=\left\langle i_{2}, i_{3}\right\rangle$ and $\phi$ is an AHI-morphism, $\phi\left(i_{2}\right)$ and $\phi\left(i_{3}\right)$ lie in $A_{M}^{\prime}$. But

$$
\phi\left(i_{2}\right)=i_{2} \phi(1)=-f_{2}+f_{3} i_{1}+f_{0} i_{2}-f_{1} i_{3} \text {, and } \phi\left(i_{3}\right)=i_{3} \phi(1)=-f_{3}-f_{2} i_{1}+f_{2} i_{2}+f_{0} i_{3} .
$$

As $\phi\left(i_{2}\right) \in A_{M}^{\prime}$, it takes values in $\mathbb{I}$, and so $f_{2}=0$. Similarly, $\phi\left(i_{3}\right)$ takes values in $\mathbb{I}$, and $f_{3}=0$. Thus $\phi(1)=f_{0}+f_{1} i_{1}$, and so $D(\phi(1))=0$ implies that $d f_{0}+I_{1} d f_{1}=0$. Therefore $\phi(1)$ takes values in $\left\langle 1, i_{1}\right\rangle$, and is holomorphic w.r.t. $I_{1}$. 


\section{Quaternionic tensor products}

In this section we will define the quaternionic tensor product of two AHI-modules $U$ and $V$, which is an AHH-module $U \otimes_{\mathbb{H}} V$. This is the key algebraic idea of this paper. In the analogy between quaternionic algebra and real algebra, AHI-modules correspond to vector spaces, and the quaternionic tensor product corresponds to the tensor product of vector spaces. The definition of the quaternionic tensor product is strange and difficult, and it is not obvious at first sight why it is a good analogue of the tensor product. This should become much clearer later on.

Definition 4.1 Let $U$ be an AHI-module. Then $\mathbb{H} \otimes\left(U^{\dagger}\right)^{*}$ is an $\mathbb{H}$-module, with $\mathbb{H}$-action $p \cdot(q \otimes x)=(p q) \otimes x$. Define a map $\iota_{U}: U \rightarrow \mathbb{H} \otimes\left(U^{\dagger}\right)^{*}$ by $\iota_{U}(u) \cdot \alpha=\alpha(u)$, for $u \in U$ and $\alpha \in U^{\dagger}$. Then $\iota_{U}$ is $\mathbb{H}$-linear, so that $\iota_{U}(U)$ is an $\mathbb{H}$-submodule of $\mathbb{H} \otimes\left(U^{\dagger}\right)^{*}$.

Suppose $u \in \operatorname{Ker} \iota_{U}$. Then $\alpha(u)=0$ for all $\alpha \in U^{\dagger}$, so that $u=0$ as $U$ is an AH-module. Thus $\iota_{U}$ is injective, and $\iota_{U}(U) \cong U$. From $(2.3)$, it follows that $\iota_{U}\left(U^{\prime}\right)=\iota_{U}(U) \cap\left(\mathbb{I} \otimes\left(U^{\dagger}\right)^{*}\right)$. Thus the AHI-module $\left(U, U^{\prime}\right)$ is determined by the $\mathbb{H}$-submodule $\iota_{U}(U)$.

Now we define the quaternionic tensor product.

Definition 4.2 Let $U, V$ be $\mathrm{A} \mathbb{H}$-modules. Then $\mathbb{H} \otimes\left(U^{\dagger}\right)^{*} \otimes\left(V^{\dagger}\right)^{*}$ is an $\mathbb{H}$-module, with $\mathbb{H}$-action $p \cdot(q \otimes x \otimes y)=(p q) \otimes x \otimes y$. Exchanging the factors of $\mathbb{H}$ and $\left(U^{\dagger}\right)^{*}$, we may regard $\left(U^{\dagger}\right)^{*} \otimes \iota_{V}(V)$ as a subspace of $\mathbb{H} \otimes\left(U^{\dagger}\right)^{*} \otimes\left(V^{\dagger}\right)^{*}$. Thus $\iota_{U}(U) \otimes\left(V^{\dagger}\right)^{*}$ and $\left(U^{\dagger}\right)^{*} \otimes \iota_{V}(V)$ are $\mathbb{H}$-submodules of $\mathbb{H} \otimes\left(U^{\dagger}\right)^{*} \otimes\left(V^{\dagger}\right)^{*}$. Define an $\mathbb{H}$-module $U \otimes_{\mathbb{H}} V$ by

$$
U \otimes_{\mathbb{H}} V=\left(\iota_{U}(U) \otimes\left(V^{\dagger}\right)^{*}\right) \cap\left(\left(U^{\dagger}\right)^{*} \otimes \iota_{V}(V)\right) \subset \mathbb{H} \otimes\left(U^{\dagger}\right)^{*} \otimes\left(V^{\dagger}\right)^{*} .
$$

Define a vector subspace $\left(U \otimes_{\mathbb{H}} V\right)^{\prime}$ by $\left(U \otimes_{\mathbb{H}} V\right)^{\prime}=\left(U \otimes_{\mathbb{H}} V\right) \cap\left(\mathbb{I} \otimes\left(U^{\dagger}\right)^{*} \otimes\left(V^{\dagger}\right)^{*}\right)$. Define a linear map $\lambda_{U, V}: U^{\dagger} \otimes V^{\dagger} \rightarrow\left(U \otimes_{\mathbb{H}} V\right)^{\times}$by $\lambda_{U, V}(x)(y)=y \cdot x \in \mathbb{H}$, for $x \in U^{\dagger} \otimes V^{\dagger}$, $y \in U \otimes_{\mathbb{H}} V$, where '.' contracts together the factors of $U^{\dagger} \otimes V^{\dagger}$ and $\left(U^{\dagger}\right)^{*} \otimes\left(V^{\dagger}\right)^{*}$.

Clearly, if $x \in U^{\dagger} \otimes V^{\dagger}$ and $y \in\left(U \otimes_{\mathbb{H}} V\right)^{\prime}$, then $\lambda_{U, V}(x)(y) \in \mathbb{I}$. As this holds for all $y \in\left(U \otimes_{\mathbb{H}} V\right)^{\prime}, \lambda_{U, V}(x) \in\left(U \otimes_{\mathbb{H}} V\right)^{\dagger}$, so that $\lambda_{U, V}$ maps $U^{\dagger} \otimes V^{\dagger} \rightarrow\left(U \otimes_{\mathbb{H}} V\right)^{\dagger}$. If $y \in U \otimes_{\mathbb{H}} V$, then $\lambda_{U, V}(x)(y)=0$ for all $x \in U^{\dagger} \otimes V^{\dagger}$ if and only if $y=0$. Thus $U \otimes_{\mathbb{H}} V$ is an AHImodule, by Definition 2.2. This AHI-module will be called the quaternionic tensor product of $U$ and $V$, and the operation $\otimes_{\mathbb{H}}$ will be called the quaternionic tensor product. When $U, V$ are finite-dimensional, $\lambda_{U, V}$ is surjective, so that $\left(U \otimes_{\mathbb{H}} V\right)^{\dagger}=\lambda_{U, V}\left(U^{\dagger} \otimes V^{\dagger}\right)$.

Here are some basic properties of the operation $\otimes_{\mathbb{H}}$.

Lemma 4.3 Let $U, V, W$ be AHI-modules. Then there are canonical AHI-isomorphisms

$$
\mathbb{H} \otimes_{\mathbb{H}} U \cong U, \quad U \otimes_{\mathbb{H}} V \cong V \otimes_{\mathbb{H}} U, \quad \text { and } \quad\left(U \otimes_{\mathbb{H}} V\right) \otimes_{\mathbb{H}} W \cong U \otimes_{\mathbb{H}}\left(V \otimes_{\mathbb{H}} W\right)
$$

Proof. As $\mathbb{H}^{\dagger} \cong \mathbb{R}$, we may identify $\mathbb{H} \otimes\left(\mathbb{H}^{\dagger}\right)^{*} \otimes\left(U^{\dagger}\right)^{*}$ and $\mathbb{H} \otimes\left(U^{\dagger}\right)^{*}$. Under this identification, it is easy to see that $\mathbb{H} \otimes_{\mathbb{H}} U$ and $\iota_{U}(U)$ are identified. Since $\iota_{U}(U) \cong U$, this gives an isomorphism

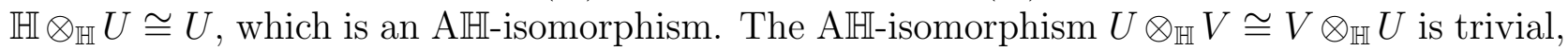
because the definition of $U \otimes_{\mathbb{H}} V$ is symmetric in $U$ and $V$. 
It remains to show that $\left(U \otimes_{\mathbb{H}} V\right) \otimes_{\mathbb{H}} W \cong U \otimes_{\mathbb{H}}\left(V \otimes_{\mathbb{H}} W\right)$. By analogy with Definition 4.2, define an $\mathbb{H}$-submodule $Z$ of $\mathbb{H} \otimes\left(U^{\dagger}\right)^{*} \otimes\left(V^{\dagger}\right)^{*} \otimes\left(W^{\dagger}\right)^{*}$ by

$$
Z=\left(\iota_{U}(U) \otimes\left(V^{\dagger}\right)^{*} \otimes\left(W^{\dagger}\right)^{*}\right) \cap\left(\left(U^{\dagger}\right)^{*} \otimes \iota_{V}(V) \otimes\left(W^{\dagger}\right)^{*}\right) \cap\left(\left(U^{\dagger}\right)^{*} \otimes\left(V^{\dagger}\right)^{*} \otimes \iota_{W}(W)\right),
$$

and define $Z^{\prime}=Z \cap\left(\mathbb{I} \otimes\left(U^{\dagger}\right)^{*} \otimes\left(V^{\dagger}\right)^{*} \otimes\left(W^{\dagger}\right)^{*}\right)$. Then $Z$ is an AHI-module. Using $\lambda_{U, V}$ and $\lambda_{U \otimes_{\mathbb{H}} V, Z}$ we may construct a map from $\left(U \otimes_{\mathbb{H}} V\right) \otimes_{\mathbb{H}} W$ to $\mathbb{H} \otimes\left(U^{\dagger}\right)^{*} \otimes\left(V^{\dagger}\right)^{*} \otimes\left(W^{\dagger}\right)^{*}$, and

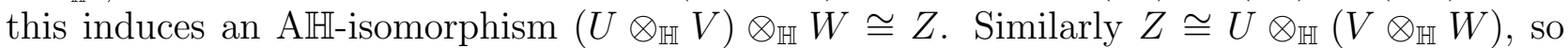
$\left(U \otimes_{\mathbb{H}} V\right) \otimes_{\mathbb{H}} W \cong U \otimes_{\mathbb{H}}\left(V \otimes_{\mathbb{H}} W\right)$, as we want.

Lemma 4.3 tells us that $\otimes_{\mathbb{H}}$ is commutative and associative, and that $\mathbb{H}$ acts as an identity element for $\otimes_{\mathbb{H}}$. Since $\otimes_{\mathbb{H}}$ is associative, we shall not bother to put brackets in multiple products such as $U \otimes_{\mathbb{H}} V \otimes_{\mathbb{H}} W$. Also, as $\otimes_{\mathbb{H}}$ is commutative and associative we can define symmetric and antisymmetric products of AHI-modules.

Definition 4.4 Let $U$ be an AHH-module. Write $\bigotimes_{\mathbb{H}}^{k} U$ for the product $U \otimes_{\mathbb{H}} \cdots \otimes_{\mathbb{H}} U$ of $k$ copies of $U$, with $\bigotimes_{\mathbb{H}}^{0} U=\mathbb{H}$. Then the $k^{\text {th }}$ symmetric group $S_{k}$ acts on $\bigotimes_{\mathbb{H}}^{k} U$ by permutation of the $U$ factors in the obvious way. Define $S_{\mathbb{H}}^{k} U$ and $\Lambda_{\mathbb{H}}^{k} U$ to be the AH-Hubmodules of $\bigotimes_{\mathbb{H}}^{k} U$ that are symmetric and antisymmetric respectively under this action of $S_{k}$.

Here is the definition of the tensor product of two AHI-morphisms.

Definition 4.5 Let $U, V, W, X$ be AHI-modules, and let $\phi: U \rightarrow W$ and $\psi: V \rightarrow X$ be AHI-morphisms. Then $\phi^{\times}\left(W^{\dagger}\right) \subset U^{\dagger}$ and $\psi^{\times}\left(X^{\dagger}\right) \subset V^{\dagger}$, by definition. Taking the duals gives maps $\left(\phi^{\times}\right)^{*}:\left(U^{\dagger}\right)^{*} \rightarrow\left(W^{\dagger}\right)^{*}$ and $\left(\psi^{\times}\right)^{*}:\left(V^{\dagger}\right)^{*} \rightarrow\left(X^{\dagger}\right)^{*}$. Combining these, we have a map

$$
\operatorname{id} \otimes\left(\phi^{\times}\right)^{*} \otimes\left(\psi^{\times}\right)^{*}: \mathbb{H} \otimes\left(U^{\dagger}\right)^{*} \otimes\left(V^{\dagger}\right)^{*} \rightarrow \mathbb{H} \otimes\left(W^{\dagger}\right)^{*} \otimes\left(X^{\dagger}\right)^{*} .
$$

Now $U \otimes_{\mathbb{H}} V \subset \mathbb{H} \otimes\left(U^{\dagger}\right)^{*} \otimes\left(V^{\dagger}\right)^{*}$ and $W \otimes_{\mathbb{H}} X \subset \mathbb{H} \otimes\left(W^{\dagger}\right)^{*} \otimes\left(X^{\dagger}\right)^{*}$. It is easy to show that $\left(\mathrm{id} \otimes\left(\phi^{\times}\right)^{*} \otimes\left(\psi^{\times}\right)^{*}\right)\left(U \otimes_{\mathbb{H}} V\right) \subset W \otimes_{\mathbb{H}} X$. Define $\phi \otimes_{\mathbb{H}} \psi: U \otimes_{\mathbb{H}} V \rightarrow W \otimes_{\mathbb{H}} X$ to be the restriction of id $\otimes\left(\phi^{\times}\right)^{*} \otimes\left(\psi^{\times}\right)^{*}$ to $U \otimes_{\mathbb{H}} V$. It follows trivially from the definitions that $\phi \otimes_{\mathbb{H}} \psi$ is $\mathbb{H}$-linear and satisfies $\left(\phi \otimes_{\mathbb{H}} \psi\right)\left(\left(U \otimes_{\mathbb{H}} V\right)^{\prime}\right) \subset\left(W \otimes_{\mathbb{H}} X\right)^{\prime}$. Thus $\phi \otimes_{\mathbb{H}} \psi$ is an AHI-morphism from $U \otimes_{\mathbb{H}} V$ to $W \otimes_{\mathbb{H}} X$. This is the quaternionic tensor product of $\phi$ and $\psi$.

Now, if $U, V$ are AHI-modules and $u \in U, v \in V$, there is in general no element ' $u \otimes_{\mathbb{H}} v$ ' in $U \otimes_{\mathbb{H}} V$ that is the product of $u$ and $v$. This is a fundamental difference between the real and quaternionic tensor products, that makes the interpretation of $U \otimes_{\mathbb{H}} V$ more difficult. However, for some special elements $u \in U, v \in V$ it is possible to define an element $u \otimes_{\mathbb{H}} v \in U \otimes_{\mathbb{H}} V$. This is shown in the following Lemma, which is trivial to prove.

Lemma 4.6 Let $U, V$ be AHH-modules, and let $u \in U$ and $v \in V$ be nonzero. Suppose that $\alpha(u) \beta(v)=\beta(v) \alpha(u) \in \mathbb{H}$ for every $\alpha \in U^{\dagger}$ and $\beta \in V^{\dagger}$. Define an element $u \otimes_{\mathbb{H}} v$ of $\mathbb{H} \otimes\left(U^{\dagger}\right)^{*} \otimes\left(V^{\dagger}\right)^{*}$ by $\left(u \otimes_{\mathbb{H}} v\right) \cdot(\alpha \otimes \beta)=\alpha(u) \beta(v) \in \mathbb{H}$. Then $u \otimes_{\mathbb{H}} v$ is a nonzero element of $U \otimes_{\mathbb{H}} V$.

\section{$5 \quad$ H-algebras and hypercomplex manifolds}

In this section we will define the quaternionic version of a commutative algebra, which we shall call an H-algebra. Then we will show that the q-holomorphic functions on a hypercomplex manifold form an H-algebra. Here is the usual definition of a commutative algebra over $\mathbb{R}$. 
Axiom A1. (i) $A$ is a real vector space.

(ii) There is a bilinear map $\mu: A \times A \rightarrow A$, called the multiplication map.

(iii) $\mu(a, b)=\mu(b, a)$ for all $a, b \in A$. Thus $\mu$ is commutative.

(iv) $\mu(\mu(a, b), c)=\mu(a, \mu(b, c))$ for all $a, b, c \in A$. Thus $\mu$ is associative.

(v) An identity $1 \in A$ is given, and $\mu(1, a)=\mu(a, 1)=a$ for all $a \in A$.

Now this axiom is not in a suitable form to translate into quaternionic language. The definition involves bilinear maps, and conditions (iii)- $(v)$ are written in terms of elements $a, b, c$ of $A$. The things we understand how to translate are tensor products and linear maps. Therefore, we rewrite the axiom in the following equivalent form, replacing bilinear maps by linear maps on a tensor product, and using linear maps rather than elements of $A$ in conditions (iii) and $(i v)$.

Axiom A2. (i) $A$ is a real vector space.

(ii) There is a linear map $\mu: A \otimes A \rightarrow A$, called the multiplication map.

(iii) $\Lambda^{2} A \subset \operatorname{Ker} \mu$. Thus $\mu$ is commutative.

(iv) The linear maps $\mu: A \otimes A \rightarrow A$ and id $: A \rightarrow A$ combine to give linear maps $\mu \otimes$ id and id $\otimes \mu: A \otimes A \otimes A \rightarrow A \otimes A$. Composing with $\mu$ gives linear maps $\mu \circ(\mu \otimes$ id $)$ and $\mu \circ($ id $\otimes \mu): A \otimes A \otimes A \rightarrow A$. Then $\mu \circ(\mu \otimes$ id $)=\mu \circ($ id $\otimes \mu)$. Thus $\mu$ is associative.

(v) An identity $1 \in A$ is given, and $\mu(1 \otimes a)=\mu(a \otimes 1)=a$ for all $a \in A$.

Now, we make a quaternionic version of Axiom A2 by replacing vector spaces by AHImodules, linear maps by AH-morphisms, and tensor products by quaternionic tensor products. Define an $\mathrm{H}$-algebra (short for Hamilton algebra) to satisfy the following axiom.

Axiom H. (i) $A$ is an AHI-module.

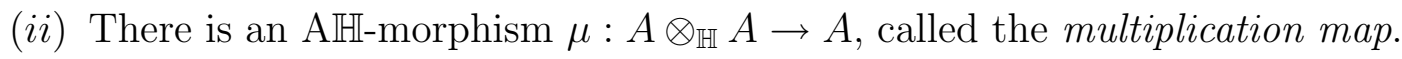

(iii) $\Lambda_{\mathbb{H}}^{2} A \subset \operatorname{Ker} \mu$. Thus $\mu$ is commutative.

(iv) The AHI-morphisms $\mu: A \otimes_{\mathbb{H}} A \rightarrow A$ and id $: A \rightarrow A$ combine to give AHImorphisms $\mu \otimes_{\mathbb{H}}$ id and id $\otimes_{\mathbb{H}} \mu: A \otimes_{\mathbb{H}} A \otimes_{\mathbb{H}} A \rightarrow A \otimes_{\mathbb{H}} A$. Composing with

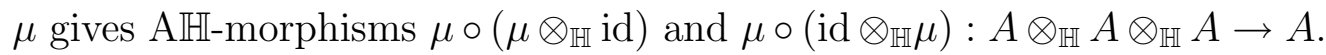
Then $\mu \circ\left(\mu \otimes_{\mathbb{H}}\right.$ id $)=\mu \circ\left(\mathrm{id} \otimes_{\mathbb{H}} \mu\right)$. Thus $\mu$ is associative.

(v) An identity $1 \in A$ is given, with $\mathbb{I} \cdot 1 \subset A^{\prime}$. This implies that if $\alpha \in A^{\dagger}$ then $\alpha(1) \in \mathbb{R}$. Thus for each $a \in A, 1 \otimes_{\mathbb{H}} a$ and $a \otimes_{\mathbb{H}} 1 \in A \otimes_{\mathbb{H}} A$ by Lemma 4.6. Then $\mu\left(1 \otimes_{\mathbb{H}} a\right)=\mu\left(a \otimes_{\mathbb{H}} 1\right)=a$ for each $a \in A$.

Here is an example.

Example 5.1 Let $U$ be an AHI-module, and define $A=\bigoplus_{j=0}^{\infty} S_{\mathbb{H}}^{j} U$. Let $\mu_{k, l}:\left(S_{\mathbb{H}}^{k} U\right) \otimes_{\mathbb{H}}$ $\left(S_{\mathbb{H}}^{l} U\right) \rightarrow S_{\mathbb{H}}^{k+l} U$ be the natural projection. The maps $\mu_{k, l}$ combine to give an AHH-morphism $\mu: A \otimes_{\mathbb{H}} A \rightarrow A$. Recall that $S_{\mathbb{H}}^{0} U=\mathbb{H}$, and define $1 \in A$ to be $1 \in S_{\mathbb{H}}^{0} U$. These make $A$ into an $\mathrm{H}$-algebra, the free $\mathrm{H}$-algebra generated by $U$.

In the next few results we will prove that if $M$ is a hypercomplex manifold, then the AHImodule $A_{M}$ of q-holomorphic functions on $M$ is an H-algebra. If $M$ and $N$ are hypercomplex manifolds, then $M \times N$ is also a hypercomplex manifold. We shall show that q-holomorphic functions on $M, N$ and $M \times N$ are related by the quaternionic tensor product $\otimes_{\mathbb{H}}$. 
Proposition 5.2 Let $M$ and $N$ be hypercomplex manifolds. Then there exists a canonical, injective AHI-morphism $\phi: A_{M} \otimes_{\mathbb{H}} A_{N} \rightarrow A_{M \times N}$.

Proof. Let $f \in A_{M} \otimes_{\mathbb{H}} A_{N}$. We will use $f$ to construct a q-holomorphic function $F$ on $M \times N$. Let $m \in M$ and $n \in N$. Then $\theta_{m} \in A_{M}^{\dagger}$ and $\theta_{n} \in A_{N}^{\dagger}$. Applying the map $\lambda_{A_{M}, A_{N}}$ of Definition $4.2, \lambda_{A_{M}, A_{N}}\left(\theta_{m} \otimes \theta_{n}\right) \in\left(A_{M} \otimes_{\mathbb{H}} A_{N}\right)^{\dagger}$. Define $F(m, n)=\lambda_{A_{M}, A_{N}}\left(\theta_{m} \otimes \theta_{n}\right) \cdot f$. This yields a $\operatorname{map} F: M \times N \rightarrow \mathbb{H}$.

As each $F$ is made from a finite number of smooth functions on $M, N$ (see below), we see that $F$ is smooth. Also, for each $n \in N$, the map $m \mapsto F(m, n)$ lies in $A_{M}$. Thus $F$ is q-holomorphic in the ' $M$ ' directions. Similarly, $F$ is q-holomorphic in the ' $N$ ' directions, so $F$ is q-holomorphic on $M \times N$, and $F \in A_{M \times N}$. Define $\phi: A_{M} \otimes_{\mathbb{H}} A_{N} \rightarrow A_{M \times N}$ by $\phi(f)=F$. Clearly $\phi$ is $\mathbb{H}$-linear. It is easy to show that $F=0$ if and only if $f=0$, and that $F \in A_{M \times N}^{\prime}$ if and only if $f \in\left(A_{M} \otimes_{\mathbb{H}} A_{N}\right)^{\prime}$. Thus $\phi$ is an injective AHI-morphism, as we have to prove.

Note that if $U, V$ are real infinite-dimensional vector spaces, then there are several ways to define the tensor product $U \otimes V$, which can give different answers. In this paper we use the convention that every element of $U \otimes V$ is a finite sum $\Sigma_{i} u_{i} \otimes v_{i}$. In the proof above, $A_{M} \otimes_{\mathbb{H}} A_{N}$ is a subspace of $\mathbb{H} \otimes\left(A_{M}^{\dagger}\right)^{*} \otimes\left(A_{N}^{\dagger}\right)^{*}$, where $\left(A_{M}^{\dagger}\right)^{*}$ and $\left(A_{N}^{\dagger}\right)^{*}$ may be infinite-dimensional. The statement that 'each $F$ is made from a finite number of smooth functions' in the proof uses the definition of $\left(A_{M}^{\dagger}\right)^{*} \otimes\left(A_{N}^{\dagger}\right)^{*}$ as a collection of finite sums.

The following Lemma is trivial, and the proof will be omitted.

Lemma 5.3 Suppose $M$ is a hypercomplex manifold, and $N$ is a hypercomplex submanifold of $M$. If $f$ is a q-holomorphic function on $M$, then $\left.f\right|_{N}$ is q-holomorphic on $N$. Let $\rho: A_{M} \rightarrow A_{N}$ be the restriction map. Then $\rho$ is an AHI-morphism.

Let $M$ be a hypercomplex manifold. Our goal is to make $A_{M}$ into an H-algebra. First we can define the multiplication map $\mu$ on $A_{M}$.

Definition 5.4 Let $M$ be a hypercomplex manifold. Proposition 5.2 gives an AHI-morphism $\phi: A_{M} \otimes_{\mathbb{H}} A_{M} \rightarrow A_{M \times M}$. Now the diagonal submanifold $\{(m, m): m \in M\}$ of $M \times M$ is a hypercomplex submanifold of $M \times M$, isomorphic to $M$. Therefore Lemma 5.3 gives an AHI-morphism $\rho: A_{M \times M} \rightarrow A_{M}$. Define an AHI-morphism $\mu: A_{M} \otimes_{\mathbb{H}} A_{M} \rightarrow A_{M}$ by $\mu=\rho \circ \phi$.

Here is the main result of this section.

Theorem 5.5 Let $M$ be a hypercomplex manifold, so that $A_{M}$ is an AHI-module. Let $1 \in A_{M}$ be the constant function on $M$ with value 1 , and let $\mu$ be the AHI-morphism given in Definition 5.4. With these definitions, $A_{M}$ is an H-algebra.

Proof. We must show that Axiom H is satisfied. Part $(i)$ holds by Definition 3.1, and parts $(i i)$ and $(v)$ are trivial. For part $(i i i)$, observe that the permutation map $A_{M} \otimes_{\mathbb{H}} A_{M} \rightarrow A_{M} \otimes_{\mathbb{H}} A_{M}$ that swaps round the factors, is induced by the map $M \times M \rightarrow M \times M$ given by $\left(m_{1}, m_{2}\right) \mapsto$ $\left(m_{2}, m_{1}\right)$. Since the diagonal submanifold is invariant under this, it follows that $\mu$ is invariant under permutation, and so $\Lambda_{\mathbb{H}}^{2} A_{M} \subset \operatorname{Ker} \mu$.

Let $\Delta_{M}^{2}$ be the 'diagonal' submanifold in $M \times M$, and let $\Delta_{M}^{3}$ be the 'diagonal' submanifold in $M \times M \times M$. We interpret part $(i v)$ as follows. $A_{M} \otimes_{\mathbb{H}} A_{M} \otimes_{\mathbb{H}} A_{M}$ is a space of q-holomorphic 
functions on $M \times M \times M$. The maps $\mu \otimes_{\mathbb{H}}$ id and id $\otimes_{\mathbb{H}} \mu$ are the maps restricting to $\Delta_{M}^{2} \times M$ and $M \times \Delta_{M}^{2}$ respectively. Thus $\mu \circ\left(\mu \otimes_{\mathbb{H}} \mathrm{id}\right)$ is the result of first restricting to $\Delta_{M}^{2} \times M$ and then to $\Delta_{M}^{3}$, and $\mu \circ\left(\mathrm{id} \otimes_{\mathbb{H}} \mu\right)$ is the result of first restricting to $M \times \Delta_{M}^{2}$ and then to $\Delta_{M}^{3}$.

Clearly $\mu \circ\left(\mu \otimes_{\mathbb{H}} \mathrm{id}\right)=\mu \circ\left(\mathrm{id} \otimes_{\mathbb{H}} \mu\right)$, proving part $(i v)$. Thus all of Axiom $\mathrm{H}$ applies, and $A_{M}$ is an H-algebra.

Theorem 5.5 is an important part of the analogy we are building between real or complex algebra and geometry, and quaternionic algebra and geometry. We know that the holomorphic functions on a complex manifold form a commutative algebra over $\mathbb{C}$, and this Theorem shows that the q-holomorphic functions on a hypercomplex manifold form an H-algebra, which is the analogue over $\mathbb{H}$ of a commutative algebra.

Next we consider the question: given an H-algebra, can we reconstruct a hypercomplex manifold from it? Let $M$ be hypercomplex and $m \in M$. Then $\theta_{m} \in A_{M}^{\dagger}$, so that $\theta_{m}: A_{M} \rightarrow \mathbb{H}$ is an AHI-morphism. But $\mathbb{H}$ itself is an $\mathrm{H}$-algebra, and $\theta_{m}$ is actually an H-algebra morphism, in the sense of the following definition:

Definition 5.6 Let $A, B$ be H-algebras, and let $\phi: A \rightarrow B$ be an AHI-morphism. Write $1_{A}, 1_{B}$ for the identities and $\mu_{A}, \mu_{B}$ for the multiplication maps in $A, B$ respectively. We say $\phi$ is an $H$-algebra morphism if $\phi\left(1_{A}\right)=1_{B}$ and $\mu_{B} \circ\left(\phi \otimes_{\mathbb{H}} \phi\right)=\phi \circ \mu_{A}$ as AHI-morphisms $A \otimes_{\mathbb{H}} A \rightarrow B$.

It is easy to see that in Lemma 5.3, the AHI-morphism $\rho$ is actually an H-algebra morphism. In the special case that $N$ is the single point $m \in M$, which is trivially a hypercomplex manifold of dimension zero, $A_{N}$ is $\mathbb{H}$ and $\rho: A_{M} \rightarrow \mathbb{H}$ is just $\theta_{m}$. This suggests a way to recover the hypercomplex manifold $M$ from the $\mathrm{H}$-algebra $A_{M}$.

Let $A$ be an $\mathrm{H}$-algebra, and define the quaternionic variety $M_{A}$ to be the set of $\mathrm{H}$-algebra morphisms $\theta: A \rightarrow \mathbb{H}$. In particularly good cases, $M_{A}$ is a manifold, with a unique hypercomplex structure determined by $A$, and $A$ is an H-subalgebra of $A_{M_{A}}$. However, the general situation is more complex, as $M_{A}$ may be singular, or may carry a different geometric structure. The study of H-algebras $A$ and their quaternionic varieties $M_{A}$ appears to be an interesting new field, which could be called hypercomplex algebraic geometry.

\section{Hyperkähler manifolds and HP-algebras}

A metric $g$ on a complex manifold $M$ is called Kähler if

$$
g_{a b}=I_{a}^{c} I_{b}^{d} g_{c d} \quad \text { and } \quad \nabla I=0
$$

where $\nabla$ is the Levi-Civita connection of $g$, and $I$ is the complex structure. The 2-form $\omega_{a c}=I_{a}^{c} g_{b c}$ is a closed $(1,1)$-form on $M$ called the Kähler form. The Kähler form $\omega$ is a symplectic form on $M$, so that $M$ is a symplectic manifold. Let $M$ be a symplectic manifold, and $P$ the algebra of smooth real functions on $M$. Then the symplectic structure on $M$ induces a bilinear map $\{\}:, P \times P \rightarrow P$ called the Poisson bracket. The algebra structure on $P$ together with the Poisson bracket $\{$,$\} make P$ into a Poisson algebra. Poisson algebras are studied in [2].

A hyperkähler structure on $M$ is a quadruple $\left(I_{1}, I_{2}, I_{3}, g\right)$, where $\left(I_{1}, I_{2}, I_{3}\right)$ is a hypercomplex structure, and $g$ is a riemannian metric that is Kähler with respect to each of $I_{1}, I_{2}$ and $I_{3}$. 
If $M$ has a hyperkähler structure, it is called a hyperkähler manifold. A hyperkähler manifold has three Kähler forms $\omega_{1}, \omega_{2}, \omega_{3}$. Hyperkähler manifolds are the natural analogue over $\mathbb{H}$ of Kähler manifolds. For more information on hyperkähler manifolds, see [13, p. 114-123], [6], [10] and [11]. If $M$ is a hyperkähler manifold, then the Kähler forms $\omega_{1}, \omega_{2}$ and $\omega_{3}$ make $M$ into a symplectic manifold in three different ways, and therefore induce three different Poisson brackets $\{,\}_{1},\{,\}_{2}$ and $\{,\}_{3}$ on the algebra $P$ of smooth real functions on $M$.

Now the previous section showed how to associate an $\mathrm{H}$-algebra $A_{M}$ with each hypercomplex manifold $M$, so that the geometry of the hypercomplex structure of $M$ is reflected in the algebraic structure of $A_{M}$. A hyperkähler structure is a hypercomplex structure with some extra data, the metric $g$. It is natural to hope that the metric $g$ on a hyperkähler manifold $M$ might be encoded in some additional algebraic information on the H-algebra $A_{M}$. One obvious possibility is that $A_{M}$ might carry some sort of quaternionic analogue of a Poisson bracket.

We shall see in this section that this is indeed true: an algebraic structure $\xi$ can be constructed on $A_{M}$ analogous to a Poisson bracket, which makes $A_{M}$ into an HP-algebra, the quaternionic analogue of a Poisson algebra. To save space, and because we have wandered from the main subject of the paper, we will omit all proofs in this section. The proofs are elementary tensor calculations and fairly dull, and we leave them as an exercise for the reader.

We start by defining a special AHI-module $Y$.

Definition 6.1 Define $Y \subset \mathbb{H}^{3}$ by $Y=\left\{\left(q_{1}, q_{2}, q_{3}\right): q_{1} i_{1}+q_{2} i_{2}+q_{3} i_{3}=0\right\}$. Then $Y \cong \mathbb{H}^{2}$ is an $\mathbb{H}$-module. Define $Y^{\prime} \subset Y$ by $Y^{\prime}=\left\{\left(q_{1}, q_{2}, q_{3}\right) \in Y: q_{j} \in \mathbb{I}\right\}$. Then $\operatorname{dim} Y^{\prime}=5$ and $\operatorname{dim} Y^{\dagger}=3$. Thus $\operatorname{dim} Y=4 j$ and $\operatorname{dim} Y^{\prime}=2 j+r$, where $j=2$ and $r=1$. Define a map $\nu: Y \rightarrow \mathbb{H}$ by $\nu\left(\left(q_{1}, q_{2}, q_{3}\right)\right)=i_{1} q_{1}+i_{2} q_{2}+i_{3} q_{3}$. Then $\operatorname{Im} \nu=\mathbb{I}$, and $\operatorname{Ker} \nu=Y^{\prime}$. But $Y / Y^{\prime} \cong\left(Y^{\dagger}\right)^{*}$, so that $\nu$ induces an isomorphism $\nu:\left(Y^{\dagger}\right)^{*} \rightarrow \mathbb{I}$. Since $\mathbb{I} \cong \mathbb{I}^{*}$, we have $\left(Y^{\dagger}\right)^{*} \cong \mathbb{I} \cong Y^{\dagger}$.

Now, define an HP-algebra, or Hamilton-Poisson algebra $A$ to satisfy Axiom $\mathrm{H}$ of $\S 5$ and Axioms $\mathrm{L}$ and $\mathrm{P}$ below. In Axiom $\mathrm{L}$, we suppose $A$ is an $\mathrm{AH}-$-module, and in Axiom $\mathrm{P}$ we suppose $A$ is an $\mathrm{H}$-algebra.

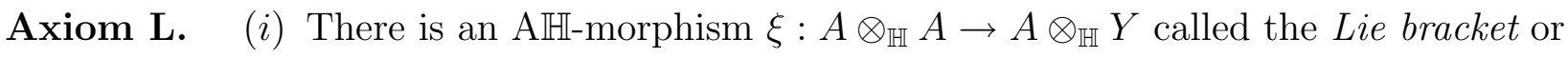
Poisson bracket, where $Y$ is the AH-module of Definition 6.1.

(ii) $S_{\mathbb{H}}^{2} A \subset \operatorname{Ker} \xi$. Thus $\xi$ is antisymmetric.

(iii) There are AHH-morphisms id $\otimes_{\mathbb{H}} \xi: A \otimes_{\mathbb{H}} A \otimes_{\mathbb{H}} A \rightarrow A \otimes_{\mathbb{H}} A \otimes_{\mathbb{H}} Y$ and $\xi \otimes_{\mathbb{H}}$ id $: A \otimes_{\mathbb{H}} A \otimes_{\mathbb{H}} Y \rightarrow A \otimes_{\mathbb{H}} Y \otimes_{\mathbb{H}} Y$. Composing gives an $\mathrm{A} \mathbb{H}-$ morphism $\left(\xi \otimes_{\mathbb{H}}\right.$ id $) \circ\left(\mathrm{id} \otimes_{\mathbb{H}} \xi\right): A \otimes_{\mathbb{H}} A \otimes_{\mathbb{H}} A \rightarrow A \otimes_{\mathbb{H}} Y \otimes_{\mathbb{H}} Y$. Then $\Lambda_{\mathbb{H}}^{3} A \subset \operatorname{Ker}\left(\left(\xi \otimes_{\mathbb{H}} \mathrm{id}\right) \circ\left(\mathrm{id} \otimes_{\mathbb{H}} \xi\right)\right)$. This is the Jacobi identity for $\xi$.

Axiom P. (i) If $a \in A$, we have $1 \otimes_{\mathbb{H}} a \in A \otimes_{\mathbb{H}} A$. Then $\xi\left(1 \otimes_{\mathbb{H}} a\right)=0$.

(ii) There are AHI-morphisms id $\otimes_{\mathbb{H}} \xi: A \otimes_{\mathbb{H}} A \otimes_{\mathbb{H}} A \rightarrow A \otimes_{\mathbb{H}} A \otimes_{\mathbb{H}} Y$ and $\mu \otimes_{\mathbb{H}}$ id $: A \otimes_{\mathbb{H}} A \otimes_{\mathbb{H}} Y \rightarrow A \otimes_{\mathbb{H}} Y$. Composing gives an AHH-morphism $\left(\mu \otimes_{\mathbb{H}}\right.$ id $) \circ\left(\mathrm{id} \otimes_{\mathbb{H}} \xi\right): A \otimes_{\mathbb{H}} A \otimes_{\mathbb{H}} A \rightarrow A \otimes_{\mathbb{H}} Y$. Similarly, there are AHImorphisms $\mu \otimes_{\mathbb{H}}$ id $: A \otimes_{\mathbb{H}} A \otimes_{\mathbb{H}} A \rightarrow A \otimes_{\mathbb{H}} A$ and $\xi: A \otimes_{\mathbb{H}} A \rightarrow A \otimes_{\mathbb{H}} Y$.

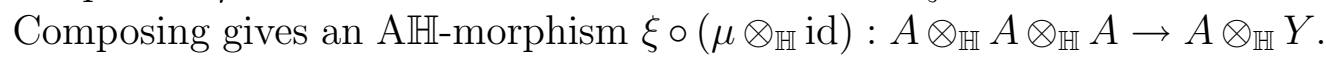
Then $\xi \circ\left(\mu \otimes_{\mathbb{H}} \mathrm{id}\right)=2\left(\mu \otimes_{\mathbb{H}} \mathrm{id}\right) \circ\left(\mathrm{id} \otimes_{\mathbb{H}} \xi\right)$ on $S_{\mathbb{H}}^{2} A \otimes_{\mathbb{H}} A$. This is the derivation property. 
Here is some motivation for these definitions. An HP-algebra is intended to be the quaternionic analogue of a Poisson algebra. Now a Poisson algebra $P$ is a commutative algebra, so its quaternionic analogue should be an H-algebra, and satisfy Axiom H. Also, a Poisson algebra has a Poisson bracket, an antisymmetric bilinear map $\{\}:, P \times P \rightarrow P$. As with the multiplication map in $\S 5$, it is convenient to rewrite this as a linear map $\xi: P \otimes P \rightarrow P$. A Poisson bracket must satisfy two important conditions. Firstly, it should satisfy the Jacobi identity, which makes into a Lie bracket on $P$. Axiom L above gives the quaternionic analogue of the definition of a Lie bracket. Secondly, the Poisson bracket and the algebra structure of $P$ must be compatible with each other in two ways: they must satisfy $\{1, a\}=0$ for all $a \in P$, and also $\{a b, c\}=a\{b, c\}+b\{a, c\}$ for all $a, b, c \in P$. This is called the derivation property. The quaternionic analogues of these conditions are given in Axiom P.

The least obvious thing about these definitions, is the use of the AHI-module $Y$. It would seem more natural for $\xi$ to map $A \otimes_{\mathbb{H}} A$ to $A$, not $A \otimes_{\mathbb{H}} Y$. The reason for this is that a hyperkähler manifold $M$ has three Poisson structures $\{,\}_{1},\{,\}_{2}$ and $\{,\}_{3}$, rather than one. We may regard $A \otimes_{\mathbb{H}} Y$ as a subspace of $A \otimes\left(Y^{\dagger}\right)^{*}$, and $\left(Y^{\dagger}\right)^{*} \cong \mathbb{I}$ by Definition 6.1. Thus $\xi$ is an antisymmetric map from $A \otimes_{\mathbb{H}} A$ to $A \otimes \mathbb{I}$, that is, a triple of antisymmetric maps from $A \otimes_{\mathbb{H}} A$ to $A$. These 3 antisymmetric maps should be interpreted as the 3 Poisson brackets on $M$.

As an example we will now construct an HP-algebra $F_{\mathfrak{g}}$ from a Lie algebra $\mathfrak{g}$. This will be useful in $\S \S 11$ and 12.

Example 6.2 Let $\mathfrak{g}$ be a Lie algebra, and let $F_{\mathfrak{g}}$ be the free H-algebra $F^{\mathfrak{g} \otimes Y}$ defined in

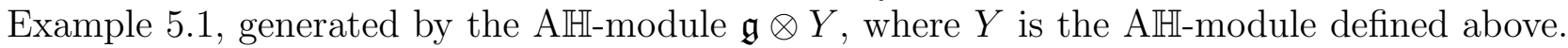
We will explain how to define an AHI-morphism $\xi: F_{\mathfrak{g}} \otimes_{\mathbb{H}} F_{\mathfrak{g}} \rightarrow F_{\mathfrak{g}} \otimes_{\mathbb{H}} Y$, that makes $F_{\mathfrak{g}}$ into an HP-algebra. Now $Y$ is a stable AHI-module in the sense of $\S 8$, and using Theorem 9.1 and Proposition 9.6 of $\S 9$, it is easy to show that $\bigotimes_{\mathbb{H}}^{j} Y=S_{\mathbb{H}}^{j} Y$. In other words, in the decomposition of $\bigotimes_{\mathbb{H}}^{j} Y$ into components under the action of the symmetric group $S_{j}$, the only nonzero component is $S_{\mathbb{H}}^{j} Y$. This implies that $S_{\mathbb{H}}^{j}(\mathfrak{g} \otimes Y)=S^{j} \mathfrak{g} \otimes S_{\mathbb{H}}^{j} Y$, and it follows that $F_{\mathfrak{g}}=\bigoplus_{j=0}^{\infty} S^{j} \mathfrak{g} \otimes S_{\mathbb{H}}^{j} Y$.

As $\mathfrak{g}$ is a Lie algebra, the Lie bracket [,] on $\mathfrak{g}$ gives a linear map $\lambda: \mathfrak{g} \otimes \mathfrak{g} \rightarrow \mathfrak{g}$, such that $\lambda(x \otimes y)=[x, y]$ for $x, y \in \mathfrak{g}$. Using $\lambda$, for $j, k \geq 1$ define $\lambda_{j, k}: S^{j} \mathfrak{g} \otimes S^{k} \mathfrak{g} \rightarrow S^{j+k-1} \mathfrak{g}$ to be the composition of maps

$$
S^{j} \mathfrak{g} \otimes S^{k} \mathfrak{g} \stackrel{\iota_{1} \otimes \iota_{2}}{\longrightarrow}\left(S^{j-1} \mathfrak{g} \otimes \mathfrak{g}\right) \otimes\left(\mathfrak{g} \otimes S^{k-1} \mathfrak{g}\right) \stackrel{\mathrm{id} \otimes \lambda \otimes \mathrm{id}}{\longrightarrow} S^{j-1} \mathfrak{g} \otimes \mathfrak{g} \otimes S^{k-1} \mathfrak{g} \stackrel{\sigma}{\longrightarrow} S^{j+k-1} \mathfrak{g},
$$

where $\iota_{1}: S^{j} \mathfrak{g} \rightarrow S^{j-1} \mathfrak{g} \otimes \mathfrak{g}$ and $\iota_{2}: S^{k} \mathfrak{g} \rightarrow \mathfrak{g} \otimes S^{k-1} \mathfrak{g}$ are the natural inclusions, and $\sigma: S^{j-1} \mathfrak{g} \otimes \mathfrak{g} \otimes S^{k-1} \mathfrak{g} \rightarrow S^{j+k-1} \mathfrak{g}$ is the symmetrization map. Define an AHI-morphism

$$
\xi_{j, k}:\left(S^{j} \mathfrak{g} \otimes S_{\mathbb{H}}^{j} Y\right) \otimes_{\mathbb{H}}\left(S^{k} \mathfrak{g} \otimes S_{\mathbb{H}}^{k} Y\right) \rightarrow\left(S^{j+k-1} \mathfrak{g} \otimes S_{\mathbb{H}}^{j+k-1} Y\right) \otimes_{\mathbb{H}} Y
$$

by $\xi_{j, k}=j k \cdot \lambda_{j, k} \otimes \mathrm{id}$, where since $S_{\mathbb{H}}^{j} Y \otimes_{\mathbb{H}} S_{\mathbb{H}}^{k} Y=S_{\mathbb{H}}^{j+k} Y=S_{\mathbb{H}}^{j+k-1} Y \otimes_{\mathbb{H}} Y$, the identity map id $: S_{\mathbb{H}}^{j} Y \otimes_{\mathbb{H}} S_{\mathbb{H}}^{k} Y \rightarrow S_{\mathbb{H}}^{j+k-1} Y \otimes_{\mathbb{H}} Y$ is a natural AHI-morphism.

Finally, define $\xi: F_{\mathfrak{g}} \otimes F_{\mathfrak{g}} \rightarrow F_{\mathfrak{g}} \otimes_{\mathbb{H}} Y$ to be $\xi_{j, k}$ on each $\left(S^{j} \mathfrak{g} \otimes S_{\mathbb{H}}^{j} Y\right) \otimes_{\mathbb{H}}\left(S^{k} \mathfrak{g} \otimes S_{\mathbb{H}}^{k} Y\right)$ in $F_{\mathfrak{g}} \otimes_{\mathbb{H}} F_{\mathfrak{g}}$. It can be shown that $F_{\mathfrak{g}}$ and $\xi$ satisfy Axioms $\mathrm{L}$ and $\mathrm{P}$ above, so that $F_{\mathfrak{g}}$ is an HP-algebra. Here the Jacobi identity $\mathrm{L}(i i i)$ for $F_{\mathfrak{g}}$ follows from the Jacobi identity satisfied by the Lie bracket $\lambda$ of the Lie algebra $\mathfrak{g}$.

Next we will explain, without proofs, how to define the quaternionic Poisson bracket $\xi$ on the H-algebra $A_{M}$ of a hyperkähler manifold. 
Definition 6.3 Let $M$ be a hyperkähler manifold. Then $M \times M$ is also a hyperkähler manifold. Let $\Delta_{M}^{2}=\{(m, m): m \in M\}$. Then $\Delta_{M}^{2}$ is a hyperkähler submanifold of $M \times M$. We shall write $M \times M=M^{1} \times M^{2}$, using the superscripts ${ }^{1}$ and ${ }^{2}$ to distinguish the two factors. Let $\nabla$ be the Levi-Civita connection on $M$. Define $\nabla^{1}, \nabla^{2}$ to be the lift of $\nabla$ to the first and second factors of $M$ in $M \times M$ respectively. Then $\nabla^{1}$ and $\nabla^{2}$ commute. Let $\nabla^{12}$ be the Levi-Civita connection on $M \times M$. Then $\nabla^{12}=\nabla^{1}+\nabla^{2}$.

Let $x \in A_{M \times M}$. Then $\nabla^{1} \nabla^{2} x \in C^{\infty}\left(\mathbb{H} \otimes T^{*} M^{1} \otimes T^{*} M^{2}\right)$ over $M \times M$. Restrict $\nabla^{1} \nabla^{2} x$ to $\Delta_{M}^{2}$. Then $\Delta_{M}^{2} \cong M$ and $\left.\left.T^{*} M^{1}\right|_{\Delta_{M}^{2}} \cong T^{*} M^{2}\right|_{\Delta_{M}^{2}} \cong T^{*} M$. Thus $\left.\nabla^{1} \nabla^{2} x\right|_{\Delta_{M}^{2}} \in C^{\infty}\left(\mathbb{H} \otimes T^{*} M \otimes\right.$ $\left.T^{*} M\right)$ over $M$. Define a linear map $\Theta: A_{M \times M} \rightarrow C^{\infty}(M, \mathbb{H}) \otimes \mathbb{I}$ by

$$
\Theta(x)=\left\{\left.g^{a b}\left(I_{1}\right)_{a}^{c} \nabla_{b}^{1} \nabla_{c}^{2} x\right|_{\Delta_{M}^{2}}\right\} \otimes i_{1}+\left\{\left.g^{a b}\left(I_{2}\right)_{a}^{c} \nabla_{b}^{1} \nabla_{c}^{2} x\right|_{\Delta_{M}^{2}}\right\} \otimes i_{2}+\left\{\left.g^{a b}\left(I_{3}\right)_{a}^{c} \nabla_{b}^{1} \nabla_{c}^{2} x\right|_{\Delta_{M}^{2}}\right\} \otimes i_{3},
$$

using index notation for tensors on $M$ in the obvious way. Here $C^{\infty}(M, \mathbb{H})$ is the space of smooth $\mathbb{H}$-valued functions on $M$.

Here are some properties of $\Theta$.

Proposition 6.4 This map satisfies $\Theta(x) \in A_{M} \otimes \mathbb{I}$. Also, $\Theta: A_{M \times M} \rightarrow A_{M} \otimes \mathbb{I}$ is an AHImorphism, and if $\Theta(x)=x_{1} \otimes i_{1}+x_{2} \otimes i_{2}+x_{3} \otimes i_{3}$ and $m \in M$, then $x_{1}(m) i_{1}+x_{2}(m) i_{2}+$ $x_{3}(m) i_{3}=0 \in \mathbb{H}$.

Now we can define the map $\xi$.

Definition 6.5 Proposition 5.2 defines an AHI-morphism $\phi: A_{M} \otimes_{\mathbb{H}} A_{M} \rightarrow A_{M \times M}$. Definition 6.3 and Proposition 6.4 define an AHI-morphism $\Theta: A_{M \times M} \rightarrow A_{M} \otimes \mathbb{I}$. Let $Y$ be the AHImodule of Definition 6.1. Then $\left(Y^{\dagger}\right)^{*} \cong \mathbb{I}$. Recall that $A_{M} \cong \iota\left(A_{M}\right)$, so we may identify $A_{M} \otimes \mathbb{I} \cong \iota\left(A_{M}\right) \otimes\left(Y^{\dagger}\right)^{*} \subset \mathbb{H} \otimes\left(A_{M}^{\dagger}\right)^{*} \otimes\left(Y^{\dagger}\right)^{*}$. Define $\xi: A_{M} \otimes_{\mathbb{H}} A_{M} \rightarrow \iota\left(A_{M}\right) \otimes\left(Y^{\dagger}\right)^{*}$ to be the composition $\xi=\Theta \circ \phi$.

Here is the main result of this section.

Theorem 6.6 This $\xi$ maps $A_{M} \otimes_{\mathbb{H}} A_{M}$ to $A_{M} \otimes_{\mathbb{H}} Y$. It is an $\mathrm{A} \mathbb{H}$-morphism, and satisfies Axioms $L$ and $P$. Thus, by Theorem 5.5, if $M$ is a hyperkähler manifold then the vector space $A_{M}$ of q-holomorphic functions on $M$ is an HP-algebra.

As with H-algebras and hypercomplex manifolds, given an HP-algebra $A$, in particularly good cases one can reconstruct a hyperkähler manifold $M$ from $A$, with its full hyperkähler structure, such that $A$ is an HP-subalgebra of $A_{M}$. Thus, hyperkähler manifolds may be constructed and studied using algebraic methods. We shall return to this idea in $\S 12$.

\section{Differences between real and quaternionic algebra}

The philosophy of this paper is that much algebra over $\mathbb{R}$ or $\mathbb{C}$ also works over $\mathbb{H}$, when we replace vector spaces by AIII-modules, and so on. However, quaternionic algebra also has properties rather unlike real or complex algebra, which come from the noncommutativity of the quaternions. In this section we discuss the differences between the theories, illustrating them by a series of examples. 
Example 7.1 Let $U, V$ be nonzero $\mathbb{H}$-modules, and let $U^{\prime}=V^{\prime}=\{0\}$. Then $U^{\dagger}=U^{\times}$and $V^{\dagger}=V^{\times}$. Suppose that $x \in U \otimes_{\mathbb{H}} V$, and let $p, q \in \mathbb{H}, \alpha \in U^{\dagger}$ and $\beta \in V^{\dagger}$. Then

$$
x \cdot(\alpha \otimes \beta) \bar{p} \bar{q}=x \cdot((p \alpha) \otimes \beta) \bar{q}=x \cdot((p \alpha) \otimes(q \beta))=x \cdot(\alpha \otimes(q \beta)) \bar{p}=x \cdot(\alpha \otimes \beta) \bar{q} \bar{p}
$$

Choosing $p$ and $q$ such that $\bar{p} \bar{q} \neq \bar{q} \bar{p}$, we see that $x \cdot(\alpha \otimes \beta)=0$. Thus $x=0$, as this holds for all $\alpha, \beta$. Therefore $U \otimes_{\mathbb{H}} V=\{0\}$, even though $U, V$ are nonzero.

Example 7.2 Let $p, q \in \mathbb{I}$ be nonzero, and let $X_{p}, X_{q}$ be the AHI-modules defined in Definition 3.2. It is easy to show that $X_{p} \otimes_{\mathbb{H}} X_{q}=\{0\}$ if $p, q$ are not proportional, and $X_{p} \otimes_{\mathbb{H}} X_{q} \cong X_{p}$ if $p, q$ are proportional. More generally, suppose $U, V$ are $\mathrm{AH}$-modules, with $\operatorname{dim} U=4 k$, $\operatorname{dim} V=4 l$. It is easy to prove that $\operatorname{dim}\left(U \otimes_{\mathbb{H}} V\right)=4 n$, where $0 \leq n \leq k l$. However, this example shows that $n$ can vary discontinuously under smooth variations of $U^{\prime}, V^{\prime}$.

These examples show that if $U$ and $V$ are nonzero AHI-modules, then $U \otimes_{\mathbb{H}} V$ may be zero, and also $\operatorname{dim}\left(U \otimes_{\mathbb{H}} V\right)$ is not well-behaved, both of which contrast with real tensor products. However, in the next two sections we will study a subclass of AHI-modules called stable AHImodules. If $U$ and $V$ are nonzero stable AHI-modules, then $U \otimes_{\mathbb{H}} V$ is nonzero, and $\operatorname{dim}\left(U \otimes_{\mathbb{H}} V\right)$ is given by a simple formula.

Let $U$ be an AHI-module with $\operatorname{dim} U=4 k$. The condition in Definition 2.2 implies that $\operatorname{dim}\left(U^{\dagger}\right) \geq k$. But $\operatorname{dim}\left(U^{\prime}\right)+\operatorname{dim}\left(U^{\dagger}\right)=4 k$, so $\operatorname{dim}\left(U^{\prime}\right) \leq 3 k$. Example 7.1 illustrates the general principle that if $\operatorname{dim}\left(U^{\prime}\right)$ is small, then quaternionic tensor products involving $U$ tend to be small or zero. A good rule is that the most interesting AHI-modules $U$ are those in the range $2 k \leq \operatorname{dim}\left(U^{\prime}\right) \leq 3 k$.

Here is another example.

Example 7.3 Define AHI-modules $U, V$ by

$$
U=\mathbb{H}^{2}, \quad U^{\prime}=\left\langle\left(i_{2}, 0\right),\left(i_{3}, 0\right),\left(1, i_{2}\right),\left(0, i_{3}\right)\right\rangle, \quad V=\mathbb{H}, \quad \text { and } \quad V^{\prime}=\left\langle i_{2}, i_{3}\right\rangle
$$

Define an AHI-morphism $\phi: U \rightarrow V$ by $\phi((p, q))=q$. A short calculation shows that $U \otimes_{\mathbb{H}} V$ and $V \otimes_{\mathbb{H}} V$ are both AHH-isomorphic to $V$, but that $\phi \otimes_{\mathbb{H}}$ id $: U \otimes_{\mathbb{H}} V \rightarrow V \otimes_{\mathbb{H}} V$ is zero.

In this example, $\phi: U \rightarrow V$ and id $: V \rightarrow V$ are both surjective, and also $\phi: U^{\prime} \rightarrow V^{\prime}$ and id $: V^{\prime} \rightarrow V^{\prime}$ are surjective, but $\phi \otimes_{\mathbb{H}}$ id $: U \otimes_{\mathbb{H}} V \rightarrow V \otimes_{\mathbb{H}} V$ is not surjective. Thus, if $\phi: U \rightarrow W$ and $\psi: V \rightarrow X$ are surjective AHI-morphisms, then $\phi \otimes_{\mathbb{H}} \psi: U \otimes_{\mathbb{H}} V \rightarrow W \otimes_{\mathbb{H}} X$ may not be surjective. In algebraic language, this means that the quaternionic tensor product $\otimes_{\mathbb{H}}$ is not right-exact. However, stable AHI-modules do satisfy a form of right-exactness, which we will not explore in this paper. The next Lemma shows that $\otimes_{\mathbb{H}}$ is left-exact.

Lemma 7.4 Suppose that $\phi: U \rightarrow W$ and $\psi: V \rightarrow X$ are injective AHI-morphisms. Then $\phi \otimes_{\mathbb{H}} \psi: U \otimes_{\mathbb{H}} V \rightarrow W \otimes_{\mathbb{H}} X$ is an injective $\mathrm{A} \mathbb{H}$-morphism.

Proof. Consider the map id $\otimes\left(\phi^{\times}\right)^{*} \otimes\left(\psi^{\times}\right)^{*}$ of $(4.4)$. Clearly this maps $\iota_{U}(U) \otimes\left(V^{\dagger}\right)^{*}$ to $\iota_{W}(W) \otimes\left(X^{\dagger}\right)^{*}$. As $\iota_{U}(U) \cong U$ and $\iota_{W}(W) \cong W$ and the map $\phi: U \rightarrow W$ is injective, we see that the kernel of id $\otimes\left(\phi^{\times}\right)^{*} \otimes\left(\psi^{\times}\right)^{*}$ on $\iota_{U}(U) \otimes\left(V^{\dagger}\right)^{*}$ is $\iota_{U}(U) \otimes \operatorname{Ker}\left(\psi^{\times}\right)^{*}$. Similarly, the kernel on $\left(U^{\dagger}\right)^{*} \otimes \iota_{V}(V)$ is $\operatorname{Ker}\left(\phi^{\times}\right)^{*} \otimes \iota_{V}(V)$. Thus the kernel of $\phi \otimes_{\mathbb{H}} \psi$ is

$$
\operatorname{Ker}\left(\phi \otimes_{\mathbb{H}} \psi\right)=\left(\iota_{U}(U) \otimes \operatorname{Ker}\left(\psi^{\times}\right)^{*}\right) \cap\left(\operatorname{Ker}\left(\phi^{\times}\right)^{*} \otimes \iota_{V}(V)\right) .
$$


But this is contained in $\left(\iota_{U}(U) \cap\left(\mathbb{H} \otimes \operatorname{Ker}\left(\phi^{\times}\right)^{*}\right)\right) \otimes\left(V^{\dagger}\right)^{*}$. Now $\iota_{U}(U) \cap\left(\mathbb{H} \otimes \operatorname{Ker}\left(\phi^{\times}\right)^{*}\right)=0$, since if $\iota(u)$ lies in $\mathbb{H} \otimes \operatorname{Ker}\left(\phi^{\times}\right)^{*}$ then $\phi(u)=0$ in $W$, so $u=0$ as $\phi$ is injective. Thus $\operatorname{Ker}\left(\phi \otimes_{\mathbb{H}} \psi\right)=0$, and $\phi \otimes_{\mathbb{H}} \psi$ is injective.

Example 7.5 Define an AHI-module $U$ by

$$
U=\mathbb{H}^{2} \quad \text { and } \quad U^{\prime}=\left\langle(1,1),\left(i_{1}, i_{2}\right),\left(i_{2}, i_{3}\right),\left(i_{3}, i_{1}\right)\right\rangle .
$$

Define $V=\{(q, 0): q \in \mathbb{H}\} \subset U$. Then $V$ is an AHI-submodule of $U$ with $V^{\prime}=\{0\}$. Put $W=U / V$, and $W^{\prime}=U^{\prime} / V^{\prime} \subset W$. But then $W$ is not an AHI-module, as the condition in Definition 2.2 is not satisfied. This example shows that the quotient of an AHI-module by an $\mathrm{AH}$-submodule is not always an AHI-module.

Here are two other differences between the quaternionic and ordinary tensor products. Firstly, despite Lemma 4.6, if $u \in U$ and $v \in V$, there is in general no element ' $u \otimes_{\mathbb{H}} v$ ' in $U \otimes_{\mathbb{H}} V$. At best, there is a real linear map from some subspace of $U \otimes V$ to $U \otimes_{\mathbb{H}} V$.

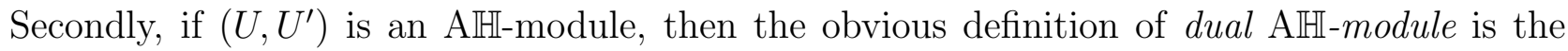
pair $\left(U^{\times}, U^{\dagger}\right)$. However, this seems not to be a fruitful idea. For $\left(U^{\times}, U^{\dagger}\right)$ may not be an AHI-module at all, and even if $U^{\times}, V^{\times}$are AHI-modules, in general $U^{\times} \otimes_{\mathbb{H}} V^{\times}$and $\left(U \otimes_{\mathbb{H}} V\right)^{\times}$ are not AHI-isomorphic.

\section{Stable and semistable AHI-modules}

Now two special sorts of AHI-modules will be defined, called stable and semistable AHI-modules. Our aim in this paper has been to develop a strong analogy between the theories of AHI-modules and vector spaces over a field. For stable AH-modules it turns out that this analogy is more complete than in the general case, because various important properties of the vector space theory hold for stable but not for general AHI-modules. Therefore, in applications of the theory it will often be useful to restrict to stable AHI-modules, to exploit their better behaviour. We begin with a definition.

Definition 8.1 We say that a finite-dimensional AHI-module $U$ is semistable if it is generated over $\mathbb{H}$ by the subspaces $U^{\prime} \cap q U^{\prime}$ for nonzero $q \in \mathbb{I}$.

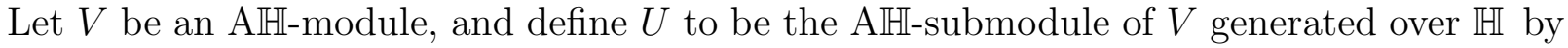
the subspaces $V^{\prime} \cap q V^{\prime}$ for nonzero $q \in \mathbb{I}$. Then $U$ is semistable, and contains all semistable AH-submodules of $V$. We call $U$ the maximal semistable AH-submodule of $V$.

Lemma 8.2 Suppose that $U$ is semistable, with $\operatorname{dim} U=4 j$ and $\operatorname{dim} U^{\prime}=2 j+r$, for integers $j, r$. Then $U^{\prime}+q U^{\prime}=U$ for generic $q \in \mathbb{I}$. Thus $r \geq 0$.

Proof. By definition, $U$ is generated over $\mathbb{H}$ by the subspaces $U^{\prime} \cap q U^{\prime}$. So suppose $U$ is generated over $\mathbb{H}$ by $U^{\prime} \cap q_{i} U^{\prime}$ for $i=1, \ldots, k$, where $0 \neq q_{i} \in \mathbb{I}$. Let $q \in \mathbb{I}$, and suppose that $q q_{i} \neq q_{i} q$ for $i=1, \ldots, k$. This is true for generic $q$. Define $W_{i}=U^{\prime} \cap q_{i} U^{\prime}$. As $U$ is generated over $\mathbb{H}$ by the $W_{i}$, we have $U=\Sigma_{i=1}^{k} \mathbb{H} \cdot W_{i}$.

Now $W_{i}=q_{i} W_{i}$, and $\mathbb{H}=\left\langle 1, q_{i}, q, q q_{i}\right\rangle$ as $q q_{i} \neq q_{i} q$. Thus $\mathbb{H} \cdot W_{i}=W_{i}+q W_{i}$. But $W_{i} \subseteq U^{\prime}$, and so $\mathbb{H} \cdot W_{i} \subseteq U^{\prime}+q U^{\prime}$. As $U=\sum_{i=1}^{k} \mathbb{H} \cdot W_{i}, U \subseteq U^{\prime}+q U^{\prime}$, so $U=U^{\prime}+q U^{\prime}$ for generic $q \in \mathbb{I}$, 
as we have to prove. Now $\operatorname{dim} U=4 j$ and $\operatorname{dim} U^{\prime}=2 j+r$, so $4 j+2 r \geq 4 j$ as $U=U^{\prime}+q U^{\prime}$, and therefore $r \geq 0$.

Next we define stable AHI-modules.

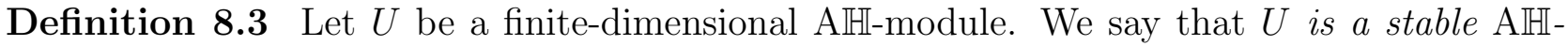
module if $U=U^{\prime}+q U^{\prime}$ for all nonzero $q \in \mathbb{I}$.

The point of this definition will become clear soon. Now let $q \in \mathbb{I}$ be nonzero. In $\S 3$ we defined an AHI-module $X_{q}$ by $X_{q}=\mathbb{H}$, and $X_{q}^{\prime}=\{p \in \mathbb{H}: p q=-q p\}$. The following properties of $X_{q}$ are easy to prove.

- $X_{q}^{\prime} \subset \mathbb{I}$ and $\operatorname{dim} X_{q}^{\prime}=\operatorname{dim} X_{q}^{\dagger}=2$.

- $X_{q}$ is semistable, but not stable.

- There is a canonical AHI-isomorphism $X_{q} \otimes_{\mathbb{H}} X_{q} \cong X_{q}$.

- Let $\chi_{q}: X_{q} \rightarrow \mathbb{H}$ be the identity map on $\mathbb{H}$. Then $\chi_{q}$ is an AHI-morphism. Hence, if $U$ is an AHH-module, then id $\otimes_{\mathbb{H}} \chi_{q}$ maps $U \otimes_{\mathbb{H}} X_{q}$ to $U \otimes_{\mathbb{H}} \mathbb{H}$. But $U \otimes_{\mathbb{H}} \mathbb{H} \cong U$, so id $\otimes_{\mathbb{H}} \chi_{q}: U \otimes_{\mathbb{H}} X_{q} \rightarrow U$ is an AHH-morphism.

- Let $U$ be an $\mathrm{A} \mathbb{H}$-module with $\operatorname{dim} U=4 j$ and $\operatorname{dim} U^{\prime}=2 j+r$. Let $q \in \mathbb{I}$ be nonzero. Now $\langle 1, q\rangle$ is a subalgebra of $\mathbb{H}$ isomorphic to $\mathbb{C}$, which acts on $U^{\prime} \cap q U^{\prime}$. Therefore $U^{\prime} \cap q U^{\prime}$ is isomorphic to $\mathbb{C}^{n}$, say, and $\operatorname{dim}\left(U^{\prime} \cap q U^{\prime}\right)=2 n$ is even.

- As $\operatorname{dim}\left(U^{\prime}+q U^{\prime}\right)=4 j+2 r-2 n$ and $U^{\prime}+q U^{\prime} \subset U$, we have $4 j+2 r-2 n \leq 4 j$, so $n \geq r$. Moreover $n=r$ if and only if $U^{\prime}+q U^{\prime}=U$.

- It can be shown that $U \otimes_{\mathbb{H}} X_{q} \cong n X_{q}$, as there is an isomorphism $\left(U \otimes_{\mathbb{H}} X_{q}\right)^{\prime} \cong U^{\prime} \cap q U^{\prime}$.

- Therefore, if $U$ is an AHI-module with $\operatorname{dim} U=4 j$ and $\operatorname{dim} U^{\prime}=2 j+r$, and $q \in \mathbb{I}$ is nonzero, then $U \otimes_{\mathbb{H}} X_{q} \cong n X_{q}$ with $n \geq r$.

If $U$ is semistable, then $U \otimes_{\mathbb{H}} X_{q} \cong r X_{q}$ for generic $q \in \mathbb{I}$, by Lemma 8.2.

Also, $U$ is stable if and only if $U \otimes_{\mathbb{H}} X_{q} \cong r X_{q}$ for all nonzero $q \in \mathbb{I}$.

Lemma 8.4 Let $V$ be a semistable AHI-module with $\operatorname{dim} V=4 k$ and $\operatorname{dim} V^{\prime}=2 k+r$. Let $U$ be the AHI-submodule of $V$ generated over $\mathbb{H}$ by the subsets $V^{\prime} \cap q V^{\prime}$ for those nonzero $q \in I$ with $V \otimes_{\mathbb{H}} X_{q} \cong r X_{q}$. Then $U$ is a stable $\mathrm{AH}$-submodule.

Proof. Clearly $U$ is semistable, by definition. As $V$ is semistable, $V \otimes_{\mathbb{H}} X_{q} \cong r X_{q}$ for generic $q \in \mathbb{I}$. But it is easily seen that if $V \otimes_{\mathbb{H}} X_{q} \cong r X_{q}$, then $U \otimes_{\mathbb{H}} X_{q} \cong r X_{q}$. Let $\operatorname{dim} U=4 j$. Then $\operatorname{dim} U^{\prime}=2 j+r$, as $U$ is semistable and $U \otimes_{\mathbb{H}} X_{q} \cong r X_{q}$ for generic $q \in \mathbb{I}$, and so $\operatorname{dim} U^{\dagger}=2 j-r$.

Suppose for a contradiction that $p \in \mathbb{I}$ is nonzero, and $U \otimes_{\mathbb{H}} X_{p} \cong n X_{p}$, with $n>r$. Write $Y=U^{\prime} \cap p U^{\prime}$, so $\operatorname{dim} Y=2 n$. Define a map $\phi: U^{\dagger} \rightarrow\left(U^{\prime}\right)^{*}$ by $\phi(\alpha) u=\operatorname{Re}(p \alpha(u))$, for $\alpha \in U^{\dagger}$ and $u \in U^{\prime}$, where $\operatorname{Re}(p \alpha(u))$ is the real part of $p \alpha(u) \in \mathbb{H}$.

Let $\alpha \in U^{\dagger}$ and $y \in Y$. Then $y \in p U^{\prime}$, so $\alpha(p y)=p \alpha(y) \in \mathbb{I}$, and $\phi(\alpha) y=0$. Thus $\phi(\alpha)$ vanishes on $Y$, and $\phi(\alpha) \in Y^{\circ} \subset\left(U^{\prime}\right)^{*}$. Therefore $\phi$ is a linear map from $U^{\dagger}$ to $Y^{\circ}$. As 
$\operatorname{dim} U^{\dagger}=2 j-r$ and $\operatorname{dim} Y^{\circ}=\operatorname{dim} U^{\prime}-\operatorname{dim} Y=2 j+r-2 n, \operatorname{dim} \operatorname{Ker} \phi \geq 2(n-r)>0$. Choose $\alpha \neq 0$ in $\operatorname{Ker} \phi$. Then $\alpha(u) \in \mathbb{I} \cap p \mathbb{I}$ for all $u \in U^{\prime}$.

Let $q \in \mathbb{I}$ satisfy $p q \neq q p$, and suppose $u \in U^{\prime} \cap q U^{\prime}$. Then $\alpha(u) \in \mathbb{I} \cap p \mathbb{I}$ and $\alpha(u) \in q \mathbb{I} \cap q p \mathbb{I}$, so $\alpha(u)=0$ and $u \in \operatorname{Ker} \alpha$. But $U$ is generated over $\mathbb{H}$ by subspaces $U^{\prime} \cap q U^{\prime}$, so $U \subset \operatorname{Ker} \alpha$, a contradiction as $\alpha \neq 0$. Therefore there exists no $p \in \mathbb{I}$ with $U \otimes_{\mathbb{H}} X_{p}=n X_{p}$ with $n>r$, and $U$ is stable.

Definition 8.5 Let $V$ be a finite-dimensional, semistable AHI-module, and let $U$ be the AHI-submodule of $V$ generated over $\mathbb{H}$ by the subspaces $V^{\prime} \cap q V^{\prime}$ for those $q \in \mathbb{I}$ for which $V^{\prime}+q V^{\prime}=V$. By Lemma 8.4, $U$ is stable, and it is easy to show that $U$ contains all stable AH-submodules of $V$. We call $U$ the maximal stable AHI-submodule of $V$.

Here are two results relating stable and semistable AHI-modules.

Theorem 8.6 All stable AHI-modules are semistable.

Proof. Let $V$ be a stable AHI-module with $\operatorname{dim} V=4 k$ and $\operatorname{dim} V^{\prime}=2 k+r$. Let $U$ be the maximal semistable AH-submodule of $V$. We will prove that $U=V$, so $V$ is semistable. Let $\operatorname{dim} U=4 j$, and let $l=k-j$. We must show $l=0$. As $U$ contains each subspace $V^{\prime} \cap q V^{\prime}$, we see that $U \otimes_{\mathbb{H}} X_{q}=V \otimes_{\mathbb{H}} X_{q} \cong r X_{q}$ for all nonzero $q \in \mathbb{I}$, so $\operatorname{dim} U^{\prime}=2 j+r$ using Lemma 8.2.

Let $W=V / U$ and $W^{\prime}=\left(V^{\prime}+U\right) / U \cong V^{\prime} / U^{\prime}$. Then $W$ is an $\mathbb{H}$-module with $\operatorname{dim} W=4 l$, and $W^{\prime}$ a real subspace with $\operatorname{dim} W^{\prime}=2 l$. Although $\left(W, W^{\prime}\right)$ need not be an AHI-module, this does not matter.

Lemma 8.7 If $l>0$, then there exists some nonzero $q \in \mathbb{I}$ with $W^{\prime} \cap q W^{\prime} \neq\{0\}$.

Proof. Let $M$ be the grassmannian of oriented real vector subspaces of $W \cong \mathbb{H}^{l}$ of dimension 2 . Then $M$ is a compact, oriented manifold of dimension $8 l-4$, which is canonically isomorphic to a nonsingular quadric $Q$ in $\mathbb{C P}^{4 l-1}$. Let $N$ be the subset of $M$ of subspaces lying in $W^{\prime}$. Then $N$ is an oriented submanifold of dimension $4 l-4$, the intersection of $Q$ with a linear subspace $\mathbb{C P}^{2 l-1}$ in $\mathbb{C} \mathbb{P}^{4 l-1}$.

Let $R$ be the subset of $W$ of 2-planes of the form $\langle w, q w\rangle$, where $w \in W$ and $q \in \mathbb{I}$ are nonzero. Then $R$ is an oriented submanifold of $W$ of dimension $4 l$, and a complex submanifold of $Q$. Now the homology classes $[N] \in H_{4 l-4}(W, \mathbb{R})$ and $[R] \in H_{4 l}(W, \mathbb{R})$ are well-defined and independent of $W^{\prime}$. Calculation shows that their intersection number is $[N] \cdot[R]=2 l$. Therefore, if $l>0$, then $N$ and $R$ must intersect, so there is some nonzero subspace $\langle w, q w\rangle \subset$ $W^{\prime}$. Thus $W^{\prime} \cap q W^{\prime} \neq\{0\}$, and the Lemma is complete.

Let $q$ be as in the Lemma. Since $\operatorname{dim} W^{\prime}=\frac{1}{2} \operatorname{dim} W$ and $W^{\prime} \cap q W^{\prime} \neq 0$, we see that $W^{\prime}+q W^{\prime} \neq W$. But the projection of $V^{\prime}+q V^{\prime}$ to $W$ lies in $W^{\prime}+q W^{\prime}$. Thus $V^{\prime}+q V^{\prime} \neq V$, a contradiction as $V$ is stable. So $l=0$, and $U=V$, and Theorem 8.6 is proved.

Proposition 8.8 Let $V$ be a finite-dimensional AHI-module. Then $V$ is semistable if and only if $V \cong U \oplus \bigoplus_{i=1}^{l} X_{q_{i}}$, where $U$ is stable and $q_{i} \in \mathbb{I}$ is nonzero. 
Proof. The 'if' part follows from Theorem 8.6 and the fact that $X_{q_{i}}$ is semistable. To prove the 'only if' part, let $V$ be semistable with $\operatorname{dim} V=4 k$ and $\operatorname{dim} V^{\prime}=2 k+r$. Let $U$ be the maximal stable AHI-module of $V$. Then $\operatorname{dim} U=4 j$ and $\operatorname{dim} U^{\prime}=2 j+r$. Let $l=k-j$. Now $V$ is semistable, which means that it is generated over $\mathbb{H}$ by $V^{\prime} \cap q V^{\prime}$ for nonzero $q \in \mathbb{I}$.

Therefore we may choose nonzero $q_{1}, \ldots, q_{l} \in \mathbb{I}$ and $v_{1}, \ldots, v_{l}$ with $v_{j} \in V^{\prime} \cap q_{j} V^{\prime}$, such that $V$ is generated over $\mathbb{H}$ by $U$ and $v_{1}, \ldots, v_{l}$. But $U$ and $v_{1}, \ldots, v_{l}$ are linearly independent over $\mathbb{H}$. Counting dimensions, we see that $V^{\prime}=U^{\prime} \oplus \bigoplus_{i=1}^{l}\left\langle v_{i}, q_{i} v_{i}\right\rangle$. Thus $V=U \oplus \bigoplus_{i=1}^{l} X_{q_{i}}$, where $X_{q_{i}}=\mathbb{H} \cdot v_{i}$, as we have to prove.

Finally we show that generic AHI-modules $\left(U, U^{\prime}\right)$ with appropriate dimensions are stable or semistable. Thus there are many stable and semistable AHI-modules.

Lemma 8.9 Let $j, r$ be integers with $0 \leq r \leq j$. Let $U=\mathbb{H}^{j}$, and let $U^{\prime}$ be a real vector subspace of $U$ with $\operatorname{dim} U^{\prime}=2 j+r$. For generic subspaces $U^{\prime},\left(U, U^{\prime}\right)$ is a semistable $\mathrm{AH}-$ module. If $r>0$, for generic subspaces $U^{\prime},\left(U, U^{\prime}\right)$ is a stable $\mathrm{A} \mathbb{H}$-module.

Proof. Let $G$ be the Grassmannian of real $(2 j+r)$-planes in $U \cong \mathbb{R}^{4 j}$. Then $U^{\prime} \in G$, and $\operatorname{dim} G=4 j^{2}-r^{2}$. The condition that $\left(U, U^{\prime}\right)$ be an AHI-module is that $\mathbb{H} \cdot U^{\dagger}=U^{\times}$. This fails for a subset of $G$ of codimension $4(j-r+1)$, so for generic $U^{\prime} \in G,\left(U, U^{\prime}\right)$ is an AHI-module. If $r=0$, it can be shown that for $U^{\prime}$ outside a subset of $G$ of codimension $2,\left(U, U^{\prime}\right) \cong \Sigma_{i=1}^{j} X_{q_{i}}$, for $q_{1}, \ldots, q_{j}$ in $\mathbb{I}$ pairwise linearly independent. As $X_{q_{i}}$ is semistable, $\left(U, U^{\prime}\right)$ is semistable for generic $U^{\prime}$.

Now suppose $r>0$, and let $0 \neq q \in \mathbb{I}$. Then the condition $U^{\prime}+q U^{\prime}=U$ fails for a subset of $G$ of codimension $2 r+2$. For $\left(U, U^{\prime}\right)$ to be stable, this condition must hold for $q \in \mathcal{S}^{2}$, the unit sphere in $\mathbb{I}$. Thus $\left(U, U^{\prime}\right)$ is stable outside a subset of $G$ of codimension $2 r$, so generic AHI-modules are stable, and hence also semistable by Theorem 8.6.

\section{Quaternionic tensor products of stable AHI-modules}

Here is the main result of this section.

Theorem 9.1 Let $U$ and $V$ be stable AHI-modules with

$$
\operatorname{dim} U=4 j, \quad \operatorname{dim} U^{\prime}=2 j+r, \quad \operatorname{dim} V=4 k, \quad \text { and } \quad \operatorname{dim} V^{\prime}=2 k+s .
$$

Then $U \otimes_{\mathbb{H}} V$ is a stable $\mathrm{AH}-$ module with $\operatorname{dim}\left(U \otimes_{\mathbb{H}} V\right)=4 l$ and $\operatorname{dim}\left(U \otimes_{\mathbb{H}} V\right)^{\prime}=2 l+t$, where $l=j s+r k-r s$ and $t=r s$.

Proof. Define $D$ to be the $\mathbb{H}$-module $\mathbb{H} \otimes\left(U^{\dagger}\right)^{*} \otimes\left(V^{\dagger}\right)^{*}$, so that $D^{\times}=\mathbb{H} \otimes U^{\dagger} \otimes V^{\dagger}$. Define $A$ to be the $\mathbb{H}$-submodule $\iota_{U}(U) \otimes\left(V^{\dagger}\right)^{*}$ of $D$, and $B$ to be the $\mathbb{H}$-submodule $\left(U^{\dagger}\right)^{*} \otimes \iota_{V}(V)$ of $D$. (These were defined in $\S 4$.) Define $C$ to be the real vector subspace $\mathbb{I} \otimes\left(U^{\dagger}\right)^{*} \otimes\left(V^{\dagger}\right)^{*}$ of D. Then

$\operatorname{dim} A=4 j(2 k-s), \operatorname{dim} B=4 k(2 j-r), \operatorname{dim} C=3(2 j-r)(2 k-s), \operatorname{dim} D=4(2 j-r)(2 k-s)$. 
Define conjugation on $D$ in the obvious way, by $\overline{(p \otimes \alpha \otimes \beta)}=\bar{p} \otimes \alpha \otimes \beta$ for $p \in \mathbb{H}$, $\alpha \in\left(U^{\dagger}\right)^{*}$ and $\beta \in\left(V^{\dagger}\right)^{*}$. Let $\bar{B}=\{\bar{b}: b \in B\}$ be the subspace of $D$ conjugate to $B$. Then $B$ is a real subspace of $D$, but not necessarily an $\mathbb{H}$-submodule. It is easy to see that

$$
A \cap B=U \otimes_{\mathbb{H}} V \quad \text { and } \quad A \cap B \cap C=A \cap \bar{B} \cap C=\left(U \otimes_{\mathbb{H}} V\right)^{\prime} .
$$

Define a subspace $K_{U, V}$ of $D^{\times}$by $z \in K_{U, V}$ if $z(\zeta)=0$ whenever $\zeta \in A$ or $\zeta \in B$. Then $K_{U, V}$ is an $\mathbb{H}$-submodule of $D^{\times}$. Clearly $\operatorname{dim} K_{U, V}=\operatorname{dim} D-\operatorname{dim}(A+B)$, and $\operatorname{dim}(A+B)=$ $\operatorname{dim} A+\operatorname{dim} B-\operatorname{dim}(A \cap B)$. As $A \cap B=U \otimes_{\mathbb{H}} V$ by (9.3), these equations and (9.2) yield $\operatorname{dim}\left(U \otimes_{\mathbb{H}} V\right)=4 l+\operatorname{dim} K_{U, V}$, where $l=j s+r k-r s$. Hence $\operatorname{dim}\left(U \otimes_{\mathbb{H}} V\right)=4 l$ if and only if $K_{U, V}=\{0\}$. So $\operatorname{dim}\left(U \otimes_{\mathbb{H}} V\right)=4 l$ by the next Lemma, as we have to prove.

Lemma 9.2 In the above, $K_{U, V}=\{0\}$.

Proof. Suppose that $W$ is an AHI-module, and $\phi: W \rightarrow V$ is an AHI-morphism. Then $\phi^{\times}: V^{\dagger} \rightarrow W^{\dagger}$, so that id $\otimes \phi^{\times}: \mathbb{H} \otimes U^{\dagger} \otimes V^{\dagger} \rightarrow \mathbb{H} \otimes U^{\dagger} \otimes W^{\dagger}$. We have $K_{U, V} \subset \mathbb{H} \otimes U^{\dagger} \otimes V^{\dagger}$ and $K_{U, W} \subset \mathbb{H} \otimes U^{\dagger} \otimes W^{\dagger}$. It is easy to show that $\left(\mathrm{id} \otimes \phi^{\times}\right)\left(K_{U, V}\right) \subset K_{U, W}$. Let $0 \neq q \in \mathbb{I}$. Set $W=V \otimes_{\mathbb{H}} X_{q}$ and let $\phi=\mathrm{id} \otimes_{\mathbb{H}} \chi_{q}: W \rightarrow V$ be the AHI-morphism defined in $\S 8$.

In this case $W \cong s X_{q}$. The argument above shows that $K_{U, X_{q}}=\{0\}$ if and only if $\operatorname{dim}\left(U \otimes_{\mathbb{H}} X_{q}\right)=4 r$. But this holds automatically, as $U$ is stable. Thus $K_{U, X_{q}}=\{0\}$, and $K_{U, W}=\{0\}$ as $W \cong s X_{q}$. Therefore $\left(\mathrm{id} \otimes \phi^{\times}\right)\left(K_{U, V}\right)=\{0\}$, so $K_{U, V} \subset \mathbb{H} \otimes U^{\dagger} \otimes \operatorname{Ker} \phi^{\times}$. Now $V$ is semistable, by Theorem 8.6. Therefore $V$ is generated by submodules $\phi(W)$ of the above type, and the intersection of the subspaces $\operatorname{Ker} \phi^{\times} \subset V^{\dagger}$ for all nonzero $q$, must be zero. So $K_{U, V} \subset \mathbb{H} \otimes U^{\dagger} \otimes\{0\}$, giving $K_{U, V}=\{0\}$, which completes the Lemma.

Next we shall study the intersection $A \cap \bar{B}$. Let $x \in \iota_{U}(U)$ and $y \in \iota_{V}(V)$. Then $x=$ $\Sigma_{e} p_{e} \otimes \alpha_{e}$, where $p_{e} \in \mathbb{H}$ and $\alpha_{e} \in\left(U^{\dagger}\right)^{*}$. Similarly $y=\Sigma_{f} q_{f} \otimes \beta_{f}$, where $q_{f} \in \mathbb{H}$ and $\beta_{f} \in\left(V^{\dagger}\right)^{*}$. Consider the element $z=\Sigma_{e, f} \bar{q}_{f} p_{e} \otimes \alpha_{e} \otimes \beta_{f}$ of $D$. Clearly $z=\Sigma_{f}\left(\bar{q}_{f} \cdot x\right) \otimes \beta_{f}$. As $\iota_{U}(U)$ is an $\mathbb{H}$-module, $\bar{q}_{f} \cdot x \in \iota_{U}(U)$, so $z \in \iota_{U}(U) \otimes\left(V^{\dagger}\right)^{*}=A$. Similarly, $\bar{z}=\Sigma_{e, f} \bar{p}_{e} q_{f} \otimes \alpha_{e} \otimes \beta_{f}$ lies in $B$. Thus $z \in A \cap \bar{B}$.

From $x \in \iota_{U}(U)$ and $y \in \iota_{V}(V)$ we have manufactured an element $z \in A \cap \bar{B}$. It is easy to see that this construction is bilinear in $x, y$, and that the set of such $z$ is a vector subspace of $A \cap \bar{B}$ of dimension $4 j k$, as $\operatorname{dim} \iota_{U}(U)=4 j$ and $\operatorname{dim} \iota_{V}(V)=4 k$. Therefore $\operatorname{dim}(A \cap \bar{B}) \geq 4 j k$. Now $\left(U \otimes_{\mathbb{H}} V\right)^{\prime}=(A \cap \bar{B}) \cap C$ by (9.3). Therefore

$$
\operatorname{dim}\left(U \otimes_{\mathbb{H}} V\right)^{\prime} \geq \operatorname{dim}(A \cap \bar{B})+\operatorname{dim} C-\operatorname{dim} D \geq 4 j k-(2 j-r)(2 k-s)=2 l+t,
$$

where $l=j s+r k-r s$ and $t=r s$.

Let $q \in \mathbb{I}$ be nonzero. Then $U \otimes_{\mathbb{H}} X_{q} \cong r X_{q}$ and $V \otimes_{\mathbb{H}} X_{q} \cong s X_{q}$, as $U, V$ are stable. Therefore $\left(U \otimes_{\mathbb{H}} V\right) \otimes_{\mathbb{H}} X_{q} \cong U \otimes_{\mathbb{H}} s X_{q} \cong r s X_{q}=t X_{q}$, using the associativity of $\otimes_{\mathbb{H}}$. It follows that $\operatorname{dim}\left(\left(U \otimes_{\mathbb{H}} V\right)^{\prime} \cap q\left(U \otimes_{\mathbb{H}} V\right)^{\prime}\right)=2 t$ for all nonzero $q \in \mathbb{I}$. However,

$$
\operatorname{dim}\left(\left(U \otimes_{\mathbb{H}} V\right)^{\prime} \cap q\left(U \otimes_{\mathbb{H}} V\right)^{\prime}\right)+\operatorname{dim}\left(\left(U \otimes_{\mathbb{H}} V\right)^{\prime}+q\left(U \otimes_{\mathbb{H}} V\right)^{\prime}\right)=2 \operatorname{dim}\left(U \otimes_{\mathbb{H}} V\right)^{\prime} .
$$

Combining these facts, the equation $\operatorname{dim}\left(U \otimes_{\mathbb{H}} V\right)=4 l$ and the inequality $\operatorname{dim}\left(U \otimes_{\mathbb{H}} V\right)^{\prime} \geq 2 l+t$, we see that $\operatorname{dim}\left(U \otimes_{\mathbb{H}} V\right)=2 l+t$, as we have to prove, and that $\left(U \otimes_{\mathbb{H}} V\right)^{\prime}+q\left(U \otimes_{\mathbb{H}} V\right)^{\prime}=U \otimes_{\mathbb{H}} V$. As this holds for all nonzero $q \in \mathbb{I}$, by definition $U \otimes_{\mathbb{H}} V$ is stable. This completes the proof of Theorem 9.1. 
Corollary 9.3 Let $U, V$ be semistable AH-modules. Then $U \otimes_{\mathbb{H}} V$ is semistable.

Proof. Write $U=W \oplus \Sigma_{i} X_{p_{i}}$ and $V=X \oplus \Sigma_{j} X_{q_{j}}$ by Proposition 8.8, where $W, X$ are stable. Now if $Y$ is any AHI-module then $Y \otimes_{\mathbb{H}} X_{q} \cong n X_{q}$ for some $n$. Therefore $U \otimes_{\mathbb{H}} V=$ $W \otimes_{\mathbb{H}} X \oplus \Sigma_{k} X_{r_{k}}$, for some collection $\left\{r_{k}\right\}$ of nonzero elements of $\mathbb{I}$. But $W \otimes_{\mathbb{H}} X$ is stable by Theorem 9.1, so $U \otimes_{\mathbb{H}} V$ is semistable by Proposition 8.8.

Thus both stable and semistable AHI-modules form subcategories of the tensor category of AII-modules, which are closed under the operations of connected sum and quaternionic tensor product. This is a useful feature, as in mathematical applications we can choose to restrict our attention to stable or semistable AHI-modules, which have better properties than general AHI-modules. The next Corollary is easy to prove.

Corollary 9.4 The dimension formulae in Theorem 9.1 also hold if $U$ is stable but $V$ is only semistable.

Now we will define the virtual dimension of an AHI-module.

Definition 9.5 Let $U$ be a stable AHH-module, with $\operatorname{dim} U=4 j$ and $\operatorname{dim} U^{\prime}=2 j+r$. Define the virtual dimension of $U$ to be $r$. If $V$ is a finite-dimensional AHH-module, let $U$ be its maximal stable AH-H-submodule, and define the virtual dimension of $V$ to be the virtual dimension of $U$.

Theorem 9.1 shows that the virtual dimension of $U \otimes_{\mathbb{H}} V$ is the product of the virtual dimensions of $U$ and $V$. Thus the virtual dimension is a good analogue of the dimension of a vector space, as it multiplies under $\otimes_{\mathbb{H}}$. Note also in Theorem 9.1 that $(j-r) / r+(k-s) / s=$ $(l-t) / t$, so that the nonnegative function $U \mapsto(j-r) / r$ behaves additively under $\otimes_{\mathbb{H}}$.

We leave the proof of the next Proposition to the reader, as a (difficult) exercise.

Proposition 9.6 Let $U$ be a stable AHI-module, with $\operatorname{dim} U=4 j$ and $\operatorname{dim} U^{\prime}=2 j+r$. Let $n$ be a positive integer. Then $S_{\mathbb{H}}^{n} U$ and $\Lambda_{\mathbb{H}}^{n} U$ are stable $\mathrm{A} \mathbb{H}-$-modules, with $\operatorname{dim}\left(S_{\mathbb{H}}^{n} U\right)=4 k$, $\operatorname{dim}\left(S_{\mathbb{H}}^{n} U\right)^{\prime}=2 k+s, \operatorname{dim}\left(\Lambda_{\mathbb{H}}^{n} U\right)=4 l$ and $\operatorname{dim}\left(\Lambda_{\mathbb{H}}^{n} U\right)^{\prime}=2 l+t$, where

$k=(j-r)\left(\begin{array}{c}r+n-1 \\ n-1\end{array}\right)+\left(\begin{array}{c}r+n-1 \\ n\end{array}\right), \quad s=\left(\begin{array}{c}r+n-1 \\ n\end{array}\right), \quad l=(j-r)\left(\begin{array}{c}r-1 \\ n-1\end{array}\right)+\left(\begin{array}{l}r \\ n\end{array}\right), \quad t=\left(\begin{array}{l}r \\ n\end{array}\right)$.

\section{Q-holomorphic functions on $\mathbb{H}$}

Let $\mathbb{H}$ have real coordinates $\left(x_{0}, \ldots, x_{3}\right)$, so that $\left(x_{0}, \ldots, x_{3}\right)$ represents $x_{0}+x_{1} i_{1}+x_{2} i_{2}+x_{3} i_{3}$. Now $\mathbb{H}$ is naturally a hypercomplex manifold with complex structures given by

$$
I_{1} d x_{2}=d x_{3}, \quad I_{2} d x_{3}=d x_{1}, \quad I_{3} d x_{1}=d x_{2} \quad \text { and } \quad I_{j} d x_{0}=d x_{j}, \quad j=1,2,3 .
$$

The study of q-holomorphic functions on $\mathbb{H}$ is called quaternionic analysis, and is surveyed in [14]. In this section, as a simple worked example of our theory, we shall study the qholomorphic polynomials on $\mathbb{H}$, finding various dimension formulae, and showing that they form an HP-algebra. 
Example 10.1 First we shall determine the AHI-module $U$ of all linear q-holomorphic functions on $\mathbb{H}$. Let $q_{0}, \ldots, q_{3} \in \mathbb{H}$, and define $u=q_{0} x_{0}+\cdots+q_{3} x_{3}$ as an $\mathbb{H}$-valued function on $\mathbb{H}$. A calculation using (10.1) shows that $u$ is q-holomorphic if and only if $q_{0}+q_{1} i_{1}+q_{2} i_{2}+q_{3} i_{3}=0$. It follows that $U \cong \mathbb{H}^{3}$. Also, $U^{\prime}$ is the vector subspace of $U$ with $q_{j} \in \mathbb{I}$ for $j=0, \ldots, 3$. Let us identify $U$ with $\mathbb{H}^{3}$ explicitly by taking $\left(q_{1}, q_{2}, q_{3}\right)$ as quaternionic coordinates. Then

$$
U^{\prime}=\left\{\left(q_{1}, q_{2}, q_{3}\right) \in \mathbb{H}^{3}: q_{j} \in \mathbb{I} \text { for } j=1,2,3 \text { and } q_{1} i_{1}+q_{2} i_{2}+q_{3} i_{3} \in \mathbb{I}\right\} .
$$

Thus $U^{\prime} \cong \mathbb{R}^{8}$, and $\operatorname{dim} U=4 j, \operatorname{dim} U^{\prime}=2 j+r$ with $j=3$ and $r=2$, so the virtual dimension of $U$ is 2 . This is because $\mathbb{H} \cong \mathbb{C}^{2}$, so the complex dimension of $\mathbb{H}$ is 2 . It is easy to see that $U$ is a stable AHI-module.

Example 10.2 Let $k \geq 0$ be an integer, and let $U^{(k)}$ be the AHH-module of q-holomorphic functions on $\mathbb{H}$ that are homogeneous polynomials of degree $k$. We shall determine $U^{(k)}$. Let $\mu: A_{\mathbb{H}} \otimes_{\mathbb{H}} A_{\mathbb{H}} \rightarrow A_{\mathbb{H}}$ be the multiplication map on $A_{\mathbb{H}}$. By Example 10.1, $U^{(1)}=U \subset A$. Thus

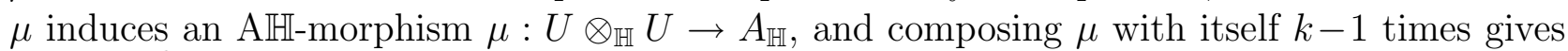
$\mu^{k-1}: \bigotimes_{\mathbb{H}}^{k} U \rightarrow A_{\mathbb{H}}$. Clearly, $\operatorname{Im} \mu^{k-1} \subset U^{(k)}$. Also, $\mu^{k-1}$ is symmetric in the $k$ factors of $U$, so it makes sense to restrict to $S_{\mathbb{H}}^{k} U$.

This gives an AHI-morphism $\mu^{k-1}: S_{\mathbb{H}}^{k} U \rightarrow U^{(k)}$. It is easily seen that $\mu^{k-1}$ is injective on $S_{\mathbb{H}}^{k} U$. By Example 10.1, $U$ is stable with $j=3$ and $r=2$. Thus Proposition 9.6 shows that $\operatorname{dim} S_{\mathbb{H}}^{k} U=2(k+1)(k+2)$. But Sudbery [14, Thm. 7, p. 217] shows that $\operatorname{dim} U^{(k)}=$ $2(k+1)(k+2)$. Therefore $\mu^{k-1}$ is an isomorphism, and $U^{(k)} \cong S_{\mathbb{H}}^{k} U$.

The interpretation of Example 10.2 is simple. If $V$ is the linear polynomials on some vector space, then $S^{k} V$ is the homogeneous polynomials of degree $k$. Here we have a quaternionic analogue of this, replacing $S^{k}$ by $S_{\mathbb{H}}^{k}$. We have found an elegant construction of the spaces $U^{(k)}$, important in quaternionic analysis, that gives insight into their algebraic structure and dimension.

Example 10.3 Let $P$ be the set of q-holomorphic polynomials on $\mathbb{H}$. Then $P=\bigoplus_{j=0}^{\infty} U^{(j)}$, by definition of $U^{(j)}$. Also, $P$ is clearly an H-subalgebra of $A_{\mathbb{H}}$, the H-algebra of q-holomorphic functions on $\mathbb{H}$. Since $U^{(j)} \cong S_{\mathbb{H}}^{j} U$ by Example 10.2, we see that $P \cong \bigoplus_{j=0}^{\infty} S_{\mathbb{H}}^{j} U$. Now Example 5.1 defined the free $H$-algebra $F^{U}$ generated by $U$, which is also given by $F^{U}=\bigoplus_{j=0}^{\infty} S_{\mathbb{H}}^{j} U$. It is easy to prove that $P$ and $F^{U}$ are isomorphic as H-algebras. The full $\mathrm{H}$-algebra $A_{\mathbb{H}}$ of q-holomorphic functions on $\mathbb{H}$ is obtained by completing $P$, by adding in convergent power series. In the same way, the $\mathrm{H}$-algebra of q-holomorphic polynomials on $\mathbb{H}^{n}$ is $F^{n U}$, the free $\mathrm{H}$-algebra generated by $n$ copies of $U$.

Now $\mathbb{H}$ is a hyperkähler manifold, so by Theorem $6.6, A_{\mathbb{H}}$ is an HP-algebra. We will define an HP-algebra structure on $P$.

Example 10.4 Let $\xi: A_{\mathbb{H}} \otimes_{\mathbb{H}} A_{\mathbb{H}} \rightarrow A_{\mathbb{H}} \otimes_{\mathbb{H}} Y$ be the Poisson bracket on $A_{\mathbb{H}}$, and consider the restriction of $\xi$ to $U^{(j)} \otimes_{\mathbb{H}} U^{(k)}$. From the definition of $\xi$, we see that $\xi$ is bilinear in the first derivatives of the two factors. The first derivatives of polynomials of degree $j, k$ are polynomials of degree $j-1, k-1$ respectively. Thus, $\xi$ must send $U^{(j)} \otimes_{\mathbb{H}} U^{(k)}$ to homogeneous polynomials of degree $j+k-2$, and so $\xi$ maps $\xi: U^{(j)} \otimes_{\mathbb{H}} U^{(k)} \rightarrow U^{(j+k-2)} \otimes_{\mathbb{H}} Y$. This implies that $\xi$ maps $\xi: P \otimes_{\mathbb{H}} P \rightarrow P \otimes_{\mathbb{H}} Y$, and so $P$ is an HP-subalgebra of $A_{\mathbb{H}}$.

In particular, consider $\xi: U \otimes_{\mathbb{H}} U \rightarrow U^{(0)} \otimes_{\mathbb{H}} Y$. Since $\xi$ is antisymmetric, we may restrict to $\Lambda_{\mathbb{H}}^{2} U$. Now $U$ is stable and has $j=3, r=2$, so by Proposition 9.6, we have $\operatorname{dim} \Lambda_{\mathbb{H}}^{2} U=8$ and 
$\operatorname{dim}\left(\Lambda_{\mathbb{H}}^{2} U\right)^{\prime}=5$. But these are the same dimensions as those of the AHH-module $Y$ of Definition 6.1. In fact there is a natural isomorphism $\Lambda_{\mathbb{H}}^{2} U \cong Y$. Now $U^{(0)} \cong \mathbb{H}$, so that $U^{(0)} \otimes_{\mathbb{H}} Y \cong Y$.

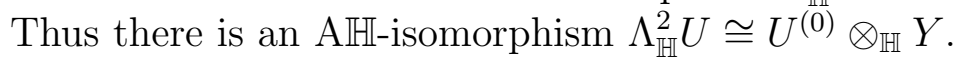

It can be shown that $\xi$ induces exactly this isomorphism $\Lambda_{\mathbb{H}}^{2} U^{(1)} \cong U^{(0)} \otimes_{\mathbb{H}} Y$. This defines $\xi$ on a generating subspace $U^{(1)}$ for $P$. Using Axiom $\mathrm{P}$ of $\xi 6$, we may extend $\xi$ uniquely to all of $P$, because the action of $\xi$ on the generators determines the whole action. This describes the HP-algebra structure of $P$.

\section{Hyperkähler manifolds with symmetry groups}

Let $M$ be a hyperkähler manifold, and suppose $v$ is a Killing vector of the hyperkähler structure on $M$. A hyperkähler moment map for $v$ is a triple $\left(f_{1}, f_{2}, f_{3}\right)$ of smooth real functions on $M$ such that $\alpha=I_{1} d f_{1}=I_{2} d f_{2}=I_{3} d f_{3}$, where $\alpha$ is the 1 -form dual to $v$ under the metric $g$. Moment maps always exist if $b^{1}(M)=0$, and are unique up to additive constants.

More generally, let $M$ be a hyperkähler manifold, let $G$ be a Lie group with Lie algebra $\mathfrak{g}$, and suppose $\rho: G \rightarrow \operatorname{Aut}(M)$ is a homomorphism from $G$ to the group of automorphisms of the hyperkähler structure on $M$. Let $\rho: \mathfrak{g} \rightarrow \operatorname{Vect}(M)$ be the induced map from $\mathfrak{g}$ to the Killing vectors. Then a hyperkähler moment map for the action $\rho$ of $G$ is a triple $\left(f_{1}, f_{2}, f_{3}\right)$ of smooth functions from $M$ to $\mathfrak{g}^{*}$, such that for each $x \in \mathfrak{g},\left(x \cdot f_{1}, x \cdot f_{2}, x \cdot f_{3}\right)$ is a hyperkähler moment map for the vector field $\rho(x)$, and in addition, $\left(f_{1}, f_{2}, f_{3}\right)$ is equivariant under the action $\rho$ of $G$ on $M$ and the coadjoint action of $G$ on $\mathfrak{g}^{*}$.

Moment maps are a familiar part of symplectic geometry, and hyperkähler moment maps were introduced by Hitchin et al. as part of a quotient construction for hyperkähler manifolds [6], [13, pp. 118-122]. Hyperkähler moment maps will exist under quite mild conditions on $M$ and $G$, for instance if $b^{1}(M)=0$ and $G$ is compact or semisimple. In this section we consider two applications of moment maps. First we will use them to construct q-holomorphic functions on hyperkähler manifolds with symmetries. Secondly, we will show that under certain conditions the moment map determines the hyperkähler structure.

Definition 11.1 Let $M$ be a hyperkähler manifold and $G$ a Lie group with Lie algebra $\mathfrak{g}$, and let $\rho: G \rightarrow \operatorname{Aut}(M)$ be an action of $G$ on $M$ preserving the hyperkähler structure. Suppose that $\left(f_{1}, f_{2}, f_{3}\right)$ is a hyperkähler moment map for $\rho$, where $f_{j}: M \rightarrow \mathfrak{g}^{*}$ is a smooth map. Define a linear map $\phi: \mathfrak{g} \otimes Y \rightarrow C^{\infty}(M, \mathbb{H})$ by

$$
\phi\left(x \otimes\left(q_{1}, q_{2}, q_{3}\right)\right)=\left(x \cdot f_{1}\right) q_{1}+\left(x \cdot f_{2}\right) q_{2}+\left(x \cdot f_{3}\right) q_{3},
$$

for each $x \in \mathfrak{g}$ and $\left(q_{1}, q_{2}, q_{3}\right) \in Y$. Here $x \cdot f_{j}$ is a smooth real function on $M$ and $q_{j} \in \mathbb{H}$, and thus $\phi\left(x \otimes\left(q_{1}, q_{2}, q_{3}\right)\right)$ is a smooth $\mathbb{H}$-valued function on $M$, and lies in $C^{\infty}(M, \mathbb{H})$.

Lemma 11.2 In the situation above, $\phi$ maps $\mathfrak{g} \otimes Y$ into the H-algebra $A_{M}$ of q-holomorphic functions on $M$, and $\phi$ is an AHI-morphism.

Proof. Define a function $y: M \rightarrow \mathbb{H}$ by $y=\left(x \cdot f_{1}\right) q_{1}+\left(x \cdot f_{2}\right) q_{2}+\left(x \cdot f_{3}\right) q_{3}$, where $x, f_{j}$ and $q_{j}$ are as in the Definition. Since $\left(f_{1}, f_{2}, f_{3}\right)$ is a hyperkähler moment map, it follows that $I_{1} d\left(x \cdot f_{1}\right)+I_{2} d\left(x \cdot f_{2}\right)+I_{3} d\left(x \cdot f_{3}\right)=0$ on $M$. Also, as $\left(q_{1}, q_{2}, q_{3}\right) \in Y$, Definition 6.1 gives that 
$q_{1} i_{1}+q_{2} i_{2}+q_{3} i_{3}=0$. These two facts together imply that $y$ is q-holomorphic on $M$, so that $\phi$ maps $\mathfrak{g} \otimes Y \rightarrow A_{M}$. As $(\mathfrak{g} \otimes Y)^{\prime}=\mathfrak{g} \otimes Y^{\prime}$, we see that $x \otimes\left(q_{1}, q_{2}, q_{3}\right)$ lies in $(\mathfrak{g} \otimes Y)^{\prime}$ if $q_{1}, q_{2}, q_{3} \in \mathbb{I}$. But then $y$ takes values in $\mathbb{I}$, and so $y \in A_{M}^{\prime}$. Thus $\phi$ maps $(\mathfrak{g} \otimes Y)^{\prime}$ into $A_{M}^{\prime}$. Since $\phi$ is clearly $\mathbb{H}$-linear, it is an AHH-morphism.

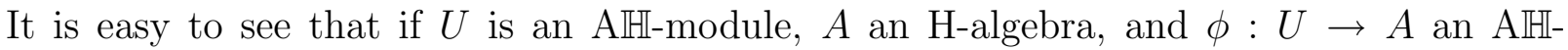
morphism, then $\phi$ extends to a unique H-algebra morphism $\Phi: F^{U} \rightarrow A$, where $F^{U}$ is the free $\mathrm{H}$-algebra generated by $U$, as in Example 5.1. Thus, Definition 11.1 and Lemma 11.2 give an H-algebra morphism $\Phi: F^{\mathfrak{g} \otimes Y} \rightarrow A_{M}$. But $A_{M}$ is an HP-algebra by Theorem 6.6 , as $M$ is hyperkähler, and Example 6.2 shows that $F^{\mathfrak{g} \otimes Y}$ is the HP-algebra $F_{\mathfrak{g}}$.

Now it can be proved that the H-algebra morphism $\Phi: F_{\mathfrak{g}} \rightarrow A_{M}$ is actually an HP-algebra morphism. The Lie group $G$ acts on $\mathfrak{g}$ by the adjoint action, and this induces an action of $G$ on $F_{\mathfrak{g}}$. Also, the action $\rho$ of $G$ on $M$ preserves the hyperkähler structure, and thus it induces an action of $G$ on $A_{M}$. So, $F_{\mathfrak{g}}$ and $A_{M}$ both come equipped with $G$-actions, which clearly preserve the HP-algebra structures. It is easy to see that the map $\Phi: F_{\mathfrak{g}} \rightarrow A_{M}$ is $G$-equivariant, that is, it commutes with the two $G$ actions. This gives the following Proposition:

Proposition 11.3 Let $M$ be a hyperkähler manifold, and $A_{M}$ the HP-algebra of q-holomorphic functions on $M$. Let $G$ be a Lie group with Lie algebra $\mathfrak{g}$, and let $\rho: G \rightarrow \operatorname{Aut}(M)$ be an action of $G$ on $M$ preserving the hyperkähler structure. Suppose $\left(f_{1}, f_{2}, f_{3}\right)$ is a hyperkähler moment map for $\rho$. Then there exists a unique HP-algebra morphism $\Phi: F_{\mathfrak{g}} \rightarrow A_{M}$, where $F_{\mathfrak{g}}$ is defined by Example 6.2, and the restriction of $\Phi$ to $\mathfrak{g} \otimes Y \subset F_{\mathfrak{g}}$ is the AHI-morphism $\phi: \mathfrak{g} \otimes Y \rightarrow A_{M}$ of Definition 11.1. Also, $\Phi$ is equivariant with respect to the natural $G$ actions on $F_{\mathfrak{g}}$ and $A_{M}$.

In our next Proposition, we show that if we know the manifold $M$ and the hyperkähler moment maps $f_{1}, f_{2}, f_{3}: M \rightarrow \mathfrak{g}^{*}$, then we can sometimes reconstruct the hyperkähler structure on $M$.

Proposition 11.4 Let $M$ be a hyperkähler manifold, $G$ a Lie group with Lie algebra $\mathfrak{g}$, and $\rho: G \rightarrow \operatorname{Aut}(M)$ an action of $G$ on $M$ preserving the hyperkähler structure. Let $\left(f_{1}, f_{2}, f_{3}\right)$ be a hyperkähler moment map for $\rho$, and define $F: M \rightarrow \mathfrak{g}^{*} \otimes \mathbb{I}$ by

$$
F(m)=f_{1}(m) \otimes i_{1}+f_{2}(m) \otimes i_{2}+f_{3}(m) \otimes i_{3}, \quad \text { for } m \in M .
$$

Suppose that at $p \in M$, the map $\left.\left.d f_{2}\right|_{p} \oplus d f_{3}\right|_{p}: T_{p} M \rightarrow \mathfrak{g}^{*} \oplus \mathfrak{g}^{*}$ is injective. Then in a neighbourhood of $p$, the hyperkähler structure of $M$ is determined solely by the functions $f_{1}, f_{2}, f_{3}$ and their first derivatives, or equivalently, by the image $F(M)$ and its tangent bundle.

Proof. We first explain how to recover the complex structure $I_{1}$ on $M$ near $p$ from the image $F(M)$. Consider the function $f_{2}+i f_{3}: M \rightarrow \mathfrak{g}^{*} \otimes \mathbb{C}$. As $\left.\left.d f_{2}\right|_{p} \oplus d f_{3}\right|_{p}$ is injective, this function embeds a neighbourhood of $p \in M$ in $\mathfrak{g}^{*} \otimes \mathbb{C}$. Now since $I_{2} d f_{2}=I_{3} d f_{3}$, we have $d f_{2}+I_{1} d f_{3}=0$, and thus $f_{2}+i f_{3}$ is holomorphic with respect to $I_{1}$. As $f_{2}+i f_{3}$ embeds $M$ near $p$, we may regard $f_{2}+i f_{3}$ as a set of holomorphic coordinates w.r.t. $I_{1}$, near $p$. But a holomorphic coordinate system determines the complex structure, and so $I_{1}$ is determined near $p$ by $F(M)$, and in fact by its tangent bundle alone.

Now let $\left(a_{1}, a_{2}, a_{3}\right),\left(b_{1}, b_{2}, b_{3}\right)$ and $\left(c_{1}, c_{2}, c_{3}\right)$ be an oriented orthonormal basis for $\mathbb{R}^{3}$. Then $\sum_{j} a_{j} I_{j}$ is a complex structure on $M$, and $\sum_{j} b_{j} f_{j}+i \sum_{j} c_{j} f_{j}$ is holomorphic with respect to 
it. Now injectivity is an open property, so if $\left(a_{1}, a_{2}, a_{3}\right)$ is sufficiently close to $(1,0,0)$ in $\mathbb{R}^{3}$, then the map $\left.\left.\sum_{j} b_{j} d f_{j}\right|_{p} \oplus \sum_{j} c_{j} d f_{j}\right|_{p}$ will be injective. Therefore by the same argument, if $\left(a_{1}, a_{2}, a_{3}\right)$ is close to $(1,0,0)$ then we can recover the complex structure $\sum_{j} a_{j} I_{j}$ from the image $F(M)$ near $p$. Thus the image $F(M)$ determines the complex structures $I_{1}, I_{2}, I_{3}$ near $p$. The metric $g$ can also be recovered using similar techniques.

\section{Classifying symmetric hyperkähler metrics}

Suppose that $M$ is a hyperkähler manifold with symmetry group $G$, and let $\Phi: F_{\mathfrak{g}} \rightarrow A_{M}$ be the HP-algebra morphism defined in Proposition 11.3. If the symmetry group $G$ is sufficiently big, in some suitable sense, then $\Phi$ contains a lot of information about $M$ and its hyperkähler structure, and we can use it to study and even classify hyperkähler manifolds with large symmetry groups. In this section we will consider hyperkähler manifolds with symmetry groups satisfying the following condition.

Condition 12.1 Let $M$ be a connected hyperkähler manifold, $G$ a Lie group, and $\rho: G \rightarrow$ $\operatorname{Aut}(M)$ an action of $G$ on $M$ preserving the hyperkähler structure, which admits a hyperkähler moment map. Then $\rho$ induces a map $\rho: \mathfrak{g} \rightarrow \operatorname{Vect}(M)$ from the Lie algebra $\mathfrak{g}$ of $G$ to the vector space $\operatorname{Vect}(M)$ of vector fields on $M$. Write $\mathcal{S}^{2}=\left\{i \in \mathbb{I}: i^{2}=-1\right\}$. For each $i=a_{1} i_{1}+a_{2} i_{2}+a_{3} i_{3} \in \mathcal{S}^{2}$, define $S_{i}$ to be the set of points $p \in M$ such that

$$
T_{p} M=\left\langle\left.\rho(x)\right|_{p}: x \in \mathfrak{g}\right\rangle+\left(a_{1} I_{1}+a_{2} I_{2}+a_{3} I_{3}\right)\left\langle\left.\rho(x)\right|_{p}: x \in \mathfrak{g}\right\rangle .
$$

Then there exists $i \in \mathcal{S}^{2}$ such that $S_{i}$ is dense in $M$.

This condition can be interpreted as follows. Let $I=a_{1} I_{1}+a_{2} I_{2}+a_{3} I_{3}$, so that $I$ is a complex structure on $M$. The condition says that for most $p \in M$, we have $T_{p} M=\left.\rho(\mathfrak{g})\right|_{p}+I\left(\left.\rho(\mathfrak{g})\right|_{p}\right)$. This means that the complexification w.r.t. $I$ of the action of $G$ is transitive near $p$, so that $M$ looks locally like an orbit of the complexified group $G^{c}$. In particular, this is only possible if $\operatorname{dim} M \leq 2 \operatorname{dim} G$, and Condition 12.1 should be interpreted as saying that the symmetry group $G$ of $M$ is 'sufficiently big'.

Proposition 12.2 Suppose that $M, G$ and $\rho$ satisfy Condition 12.1. Let $F: M \rightarrow \mathfrak{g}^{*} \otimes \mathbb{I}$ be defined by (11.2). Then $F$ is a smooth map, and there is a dense open set $S_{i} \in M$ such that the map $F: S_{i} \rightarrow \mathfrak{g}^{*} \otimes \mathbb{I}$ is an immersion, and the hyperkähler structure on $S_{i}$ is determined by its image $F\left(S_{i}\right)$.

Proof. By Condition 12.1, there exists some $i \in \mathcal{S}^{2}$ such that the set $S_{i}$ is dense in $M$. Now $S_{i}$ is clearly open, by its definition, and $F$ is smooth as each $f_{j}$ is smooth. Suppose for simplicity that $i=i_{1}$, since the proof for general $i \in \mathcal{S}^{2}$ follows from an $S O(3)$ rotation in $\mathbb{I}$. Let $p \in S_{i_{1}}$, so that $T_{p} M$ is spanned by the vectors $\left.\rho(x)\right|_{p}$ and $\left.I_{1} \rho(x)\right|_{p}$, for $x \in \mathfrak{g}$. Contracting these vectors with the Kähler form $\omega_{2}$, we see that $T_{p}^{*} M$ is spanned by the 1 -forms $\left.\rho(x) \cdot \omega_{2}\right|_{p}$ and $\left.\left(I_{1} \rho(x)\right) \cdot \omega_{2}\right|_{p}$. But $\rho(x) \cdot \omega_{2}=d f_{2}$ by definition of $f_{2}$, and similarly $\left(I_{1} \rho(x)\right) \cdot \omega_{2}=-\rho(x) \cdot \omega_{3}=-x \cdot d f_{3}$. Thus $T_{p}^{*} M$ is spanned by the 1 -forms $\left.x \cdot d f_{2}\right|_{p}$ and $\left.x \cdot d f_{3}\right|_{p}$ for $x \in \mathfrak{g}$.

Therefore, an alternative definition of the set $S_{i_{1}}$ is that $p \in S_{i_{1}}$ if and only if the map $\left.\left.d f_{2}\right|_{p} \oplus d f_{3}\right|_{p}: T_{p} M \rightarrow \mathfrak{g}^{*} \oplus \mathfrak{g}^{*}$ is injective. We immediately deduce that $F$ is an immersion on 
$S_{i_{1}}$, and Proposition 11.4 applies to show that the hyperkähler structure on $S_{i_{1}}$ is determined by the image $F\left(S_{i_{1}}\right)$. This completes the proof.

From Proposition 11.3, $G$ acts on $F_{\mathfrak{g}}$ and $A_{M}$. Write $F_{\mathfrak{g}}^{G}$ and $A_{M}^{G}$ for the $G$-invariant subspaces of $F_{\mathfrak{g}}$ and $A_{M}$ respectively. Then

$$
F_{\mathfrak{g}}^{G}=\bigoplus_{j=0}^{\infty}\left(S^{j} \mathfrak{g}\right)^{G} \otimes S_{\mathbb{H}}^{j} Y,
$$

and $F_{\mathfrak{g}}^{G}$ and $A_{M}^{G}$ are HP-subalgebras of $F_{\mathfrak{g}}$ and $A_{M}$. In fact, it can be shown that the Poisson bracket $\xi$ of $F_{\mathfrak{g}}$ vanishes on $F_{\mathfrak{g}}^{G}$, so only the $\mathrm{H}$-algebra structure is nontrivial. Since $\Phi: F_{\mathfrak{g}} \rightarrow$ $A_{M}$ is $G$-equivariant, it follows that $\Phi$ takes $F_{\mathfrak{g}}^{G}$ to $A_{M}^{G}$. But if Condition 12.1 holds, then $\Phi$ takes $F_{\mathfrak{g}}^{G}$ to the constant functions, as we will now show.

Proposition 12.3 Suppose that $M, G$ and $\rho$ satisfy Condition 12.1. Then the map $\Phi: F_{\mathfrak{g}}^{G} \rightarrow$ $A_{M}^{G}$ takes each $y \in F_{\mathfrak{g}}^{G}$ to a constant function on $M$.

Proof. By (12.2), it is sufficient to prove the result for $y \in\left(S^{j} \mathfrak{g}\right)^{G} \otimes S_{\mathbb{H}}^{j} Y$. Write $U=\left(S^{j} \mathfrak{g}\right)^{G} \otimes$ $S_{\mathbb{H}}^{j} Y$. Now $Y$ is stable, in the sense of $\S 8$. By Proposition 9.6, $S_{\mathbb{H}}^{j} Y$ is also stable, and by Theorem 8.6 it is semistable. Thus $U$ is semistable, so that $U$ is generated over $\mathbb{H}$ by the subspaces $U^{\prime} \cap q U^{\prime}$ for nonzero $q \in \mathbb{I}$. But we can say more: because $U$ is stable, the subspace $U^{\prime} \cap q U^{\prime}$ for nonzero $q$ depends real-analytically on $q$ (in particular, the dimension remains constant). Because of this, one can show that $U$ is generated over $\mathbb{H}$ by the subspaces $U^{\prime} \cap i U^{\prime}$ for $i$ in any given nonempty open set $T \subset \mathcal{S}^{2}$.

Define $T \subset \mathcal{S}^{2}$ to be the set of $i \in \mathcal{S}^{2}$ such that the set $S_{i}$ defined in Condition 12.1 is dense in $M$. It can be shown using transversality arguments that $T$ is open in $\mathcal{S}^{2}$, and $T$ contains at least one element by Condition 12.1, so $T$ is nonempty. Let $i \in T$, and suppose that $y \in U^{\prime} \cap i U^{\prime}$. We will show that $\Phi(y)$ is constant on $M$.

Again we may suppose that $i=i_{1}$, as the proof for general $i$ follows by an $S O(3)$ rotation of $\mathbb{I}$. Write

$$
\Phi(y)=f_{0}+f_{1} i_{1}+f_{2} i_{2}+f_{3} i_{3},
$$

where $f_{0}, \ldots, f_{3}$ are real functions on $M$. Now $y \in U^{\prime}$. Thus $\Phi(y) \in A_{M}^{\prime}$, so that $\Phi(y)$ takes values in $\mathbb{I}$, and therefore $f_{0}=0$. Similarly, $y \in i_{1} U^{\prime}$, so $i_{1} \Phi(y)$ takes values in $\mathbb{I}$ and $f_{1}=0$, giving $\Phi(y)=f_{2} i_{2}+f_{3} i_{3}$. Since $\Phi(y)$ is q-holomorphic, we see that $d f_{2}=I_{1} d f_{3}$ on $M$.

Now $\Phi(y) \in A_{M}^{G}$, so $f_{2}$ and $f_{3}$ are $G$-invariant. Thus, $d f_{2}$ and $d f_{3}$ give zero when contracted with the vector fields $\rho(x)$ for $x \in \mathfrak{g}$. But $d f_{2}=I_{1} d f_{3}$, and so $d f_{2}$ and $d f_{3}$ also give zero when contracted with the vector fields $I_{1} \rho(x)$ for $x \in \mathfrak{g}$. By (12.1) these vector fields $\operatorname{span} T_{p} M$ for $p \in S_{i_{1}}$, so $d f_{2}=d f_{3}=0$ on $S_{i_{1}}$. But $S_{i_{1}}$ is dense in $M$ by definition of $T$, so $d f_{2}=d f_{3}=0$ on $M$ by continuity, and as $M$ is connected, $f_{2}$ and $f_{3}$ are constant on $M$. Therefore $\Phi(y)$ is constant.

We have shown that if $y \in U^{\prime} \cap i U^{\prime}$ for $i \in T$, then $\Phi(y)$ is constant. But $T$ is dense and nonempty, and so $U$ is generated over $\mathbb{H}$ by such subspaces $U^{\prime} \cap i U^{\prime}$. Thus $\Phi(y)$ is constant for $y$ in a subset that generates $U$ over $\mathbb{H}$, so $\Phi(y)$ is constant for all $y \in U$. This completes the proof.

The Proposition shows that $\Phi$ maps $F_{\mathfrak{g}}^{G}$ to $\mathbb{H} \subset A_{M}$, the constant functions in $A_{M}$. Now $\mathbb{H}$ is an H-subalgebra of $A_{M}$, and $\Phi: F_{\mathfrak{g}}^{G} \rightarrow \mathbb{H}$ is an H-algebra morphism. This morphism actually 
contains a lot of information about the geometry of $M$, so much so that it is possible locally to reconstruct the manifold $M$ with its hyperkähler structure from the H-algebra morphism $\Phi: F_{\mathfrak{g}}^{G} \rightarrow \mathbb{H}$. Here is the first step in this reconstruction.

Definition 12.4 Let $G$ be a Lie group, with Lie algebra $\mathfrak{g}$. Then $F_{\mathfrak{g}}$ and $F_{\mathfrak{g}}^{G}$ are H-algebras. As $Y^{\dagger} \cong \mathbb{I}$, we have $(\mathfrak{g} \otimes Y)^{\dagger} \cong \mathfrak{g}^{*} \otimes \mathbb{I}$. Thus each $m \in \mathfrak{g}^{*} \otimes \mathbb{I}$ determines an AIHI-morphism $\psi_{m}$ : $\mathfrak{g} \otimes Y \rightarrow \mathbb{H}$. Since $F_{\mathfrak{g}}$ is the free $\mathrm{H}$-algebra $F^{\mathfrak{g} \otimes Y}$, this extends to a unique H-algebra morphism $\Psi_{m}: F_{\mathfrak{g}} \rightarrow \mathbb{H}$. Clearly, the restriction of $\Psi_{m}$ to $F_{\mathfrak{g}}^{G}$ is an H-algebra morphism $\Psi_{m}: F_{\mathfrak{g}}^{G} \rightarrow \mathbb{H}$.

Now let $\Phi: F_{\mathfrak{g}}^{G} \rightarrow \mathbb{H}$ be a given H-algebra morphism. Define $M_{\Phi}$ to be the subset of $m \in \mathfrak{g}^{*} \otimes \mathbb{I}$ such that the restriction of $\Psi_{m}$ to $F_{\mathfrak{g}}^{G}$ is equal to $\Phi$. It is easy to show (using Hilbert's Nullstellensatz) that $M_{\Phi}$ is the zeros of a finite number of polynomials on $\mathfrak{g}^{*} \otimes \mathbb{I}$, so that $M_{\Phi}$ is a real algebraic variety, and is a manifold with singularities.

Let $M, G, \rho$ and $\Phi$ be as in the previous Proposition. Then $\Phi: F_{\mathfrak{g}}^{G} \rightarrow \mathbb{H}$ is an H-algebra morphism, so that Definition 12.4 defines a variety $M_{\Phi}$ in $\mathfrak{g}^{*} \otimes \mathbb{I}$. The next result explains the relation between $M$ and $M_{\Phi}$.

Proposition 12.5 Suppose that $M, G$ and $\rho$ satisfy Condition 12.1. Let $\Phi: F_{\mathfrak{g}}^{G} \rightarrow \mathbb{H}$ be the $H$-algebra morphism defined above, and let $F: M \rightarrow \mathfrak{g}^{*} \otimes \mathbb{I}$ be defined by (11.2). Then the image $F(M)$ is a subset of the real algebraic variety $M_{\Phi}$ defined in Definition 12.4.

Proof. Let $m \in M$. Then Definition 3.1 defines an H-algebra morphism $\theta_{m}: A_{M} \rightarrow \mathbb{H}$. Thus $\theta_{m} \circ \phi: \mathfrak{g} \otimes Y \rightarrow \mathbb{H}$ is an AHI-morphism, and $\theta_{m} \circ \phi \in(\mathfrak{g} \otimes Y)^{\dagger} \cong \mathfrak{g}^{*} \otimes \mathbb{I}$. Let $p$ be the element of $\mathfrak{g}^{*} \otimes \mathbb{I}$ corresponding to $\theta_{m} \circ \phi$. It follows easily from the definitions that $F(m)=p$, and $\psi_{p}=\theta_{m} \circ \phi$, and $\Psi_{p}=\theta_{m} \circ \Phi$. Consider the restriction of $\theta_{m} \circ \Phi$ to $F_{\mathfrak{g}}^{G}$. If $y \in F_{\mathfrak{g}}^{G}$ then $\Phi(y)$ is constant on $M$. But $\theta_{m} \circ \Phi(y)$ just evaluates $\Phi(y)$ at $m$, so identifying $\mathbb{H}$ with the constant $\mathbb{H}$-valued functions on $M$ gives $\theta_{m} \circ \Phi(y)=\Phi(y)$. Thus $\Psi_{p}=\theta_{m} \circ \Phi=\Phi$ on $F_{\mathfrak{g}}^{G}$, and $p \in M_{\Phi}$ by definition. But $p=F(m)$, so $F(m) \in M_{\Phi}$ for each $m \in M$, and $F(M)$ is a subset of $M_{\Phi}$, as we have to prove.

It can be shown, although we will not prove it, that if $\mathfrak{g}$ is semisimple then $\operatorname{dim} M_{\Phi}=$ $2 \operatorname{dim} \mathfrak{g}-2 \operatorname{rank} \mathfrak{g}$, for every H-algebra morphism $\Phi: F_{\mathfrak{g}}^{G} \rightarrow \mathbb{H}$. Thus, if $\operatorname{dim} M=2 \operatorname{dim} \mathfrak{g}-$ 2 rank $\mathfrak{g}$, then $F(M)$ is an open subset of $M_{\Phi}$, at least away from the singularities of $M_{\Phi}$. In this case, locally $F(M)$ is equal to $M_{\Phi}$. But Proposition 12.2 shows that the image $F(M)$ determines the hyperkähler structure of $M$ in a dense subset, and hence on all of $M$ by continuity. This proves:

Corollary 12.6 Suppose that $M, G$ and $\rho$ satisfy Condition 12.1, and suppose that $G$ is semisimple and $\operatorname{dim} M=2 \operatorname{dim} \mathfrak{g}-2 \operatorname{rank} \mathfrak{g}$. Then the hyperkähler structure of $M$ is determined by the real algebraic variety $M_{\Phi}$, and hence by the H-algebra morphism $\Phi: F_{\mathfrak{g}}^{G} \rightarrow \mathbb{H}$.

This result suggests that given a Lie group $G$, if $\Phi: F_{\mathfrak{g}}^{G} \rightarrow \mathbb{H}$ is an H-algebra morphism, then we should be able to construct a $G$-invariant hyperkähler metric on some open subset of the variety $M_{\Phi}$ of Definition 12.4. In fact this is true in many cases, although the author has not proved that a hyperkähler metric exists on every $M_{\Phi}$. Thus, for each Lie group $G$ one may construct a natural family of hyperkähler manifolds with symmetry group $G$. Here is a simple example. 
Example 12.7 Let $G$ be $S O(3)$, so that $\mathfrak{g}=\mathfrak{s o}(3)$. Then $\mathfrak{g}$ has a basis $v_{1}, v_{2}, v_{3}$ with

$$
\left[v_{1}, v_{2}\right]=v_{3}, \quad\left[v_{2}, v_{3}\right]=v_{1}, \quad \text { and } \quad\left[v_{3}, v_{1}\right]=v_{2}
$$

Consider the element $\alpha=v_{1} \otimes v_{1}+v_{2} \otimes v_{2}+v_{3} \otimes v_{3}$ in $S^{2} \mathfrak{g}$. It is easy to show that $\alpha$ is invariant under the action of $G$ on $S^{2} \mathfrak{g}$, so that $\alpha \in\left(S^{2} \mathfrak{g}\right)^{G}$, and in fact $\left(S^{2} \mathfrak{g}\right)^{G}=\langle\alpha\rangle$. Thus $\langle\alpha\rangle \otimes S_{\mathbb{H}}^{2} Y \subset F_{\mathfrak{g}}^{G}$. Write $U=\langle\alpha\rangle \otimes S_{\mathbb{H}}^{2} Y$, so that $U$ is an AHI-module of $F_{\mathfrak{g}}^{G}$ isomorphic to $S_{\mathbb{H}}^{2} Y$. It turns out that $U$ generates $F_{\mathfrak{g}}^{G}$ as an H-algebra, and $F_{\mathfrak{g}}^{G}$ is isomorphic as an H-algebra to $F^{U}$, the free $\mathrm{H}$-algebra generated by $U$.

Now there is a 1-1 correspondence between AHI-morphisms $\phi: U \rightarrow \mathbb{H}$ and H-algebra morphisms $\Phi: F^{U} \rightarrow \mathbb{H}$. But AHI-morphisms $\phi: U \rightarrow \mathbb{H}$ are just elements of $U^{\dagger}$. As $U \cong S_{\mathbb{H}}^{2} Y$, and $Y$ is stable with $\operatorname{dim} Y=8$ and $\operatorname{dim} Y^{\prime}=5$, by Theorem 9.6 we have $\operatorname{dim} U=12$ and $\operatorname{dim} U^{\prime}=7$, giving $\operatorname{dim} U^{\dagger}=5$. Thus, there is a 1-1 correspondence between H-algebra morphisms $\Phi: F_{\mathfrak{g}}^{G} \rightarrow \mathbb{H}$, and elements $\phi \in U^{\dagger} \cong \mathbb{R}^{5}$.

There is a natural identification between $U^{\dagger}=\mathbb{R}^{5}$ and symmetric trace-free $3 \times 3$ matrices. Thus we may identify $\phi \in U^{\dagger}$ with the matrix $\left(a_{i j}\right)$, where $a_{i j}=a_{j i}$ and $a_{11}+a_{22}+a_{33}=0$. If $\Phi$ is the corresponding H-algebra morphism $\Phi: F_{\mathfrak{g}}^{G} \rightarrow \mathbb{H}$, then it is easy to show that the subset $M_{\Phi}$ of $\mathfrak{g}^{*} \otimes \mathbb{I}$ defined by Definition 12.4 is the set of elements $\mathbf{r}_{1} \otimes i_{1}+\mathbf{r}_{2} \otimes i_{2}+\mathbf{r}_{3} \otimes i_{3}$ in $\mathfrak{g}^{*} \otimes \mathbb{I}$ satisfying the five equations

$$
\mathbf{r}_{1} \cdot \mathbf{r}_{2}=a_{12}, \quad \mathbf{r}_{1} \cdot \mathbf{r}_{3}=a_{13}, \quad \mathbf{r}_{2} \cdot \mathbf{r}_{3}=a_{23}, \quad \mathbf{r}_{1} \cdot \mathbf{r}_{1}-a_{11}=\mathbf{r}_{2} \cdot \mathbf{r}_{2}-a_{22}=\mathbf{r}_{3} \cdot \mathbf{r}_{3}-a_{33}
$$

where the scalar product on $\mathfrak{g}^{*}$ is given by the Killing form. These are five equations in the nine variables of $\mathbf{r}_{1}, \mathbf{r}_{2}, \mathbf{r}_{3}$, so that $M_{\Phi}$ has dimension 4 . Since $\operatorname{dim} \mathfrak{g}=3$ and rank $\mathfrak{g}=1$, the dimension is $2 \operatorname{dim} \mathfrak{g}-2 \operatorname{rank} \mathfrak{g}$, as we claimed above.

Careful investigation shows that following the methods of Proposition 11.4, we can reconstruct a unique hyperkähler structure on a dense open set of $M_{\Phi}$, for every such matrix $\left(a_{i j}\right)$. Then Corollary 12.6 shows that every $S O(3)$-invariant hyperkähler structure on a 4-manifold $M$ satisfying Condition 12.1 is locally isomorphic to the hyperkähler structure on some $M_{\Phi}$. Now in [1], Belinskii et al. explicitly determine all hyperkähler metrics with an $S O(3)$-action of this form by solving an ODE, so our metrics must correspond to theirs.

Applying an $S O(3)$ rotation to $\mathbb{I}$ has the effect of conjugating $\left(a_{i j}\right)$ by this matrix. Therefore, after an $S O(3)$ rotation in $\mathbb{I}$, we may suppose that $\left(a_{i j}\right)$ is diagonal with $a_{11} \geq a_{22} \geq a_{33}$. Equation (12.5) then becomes

$$
\mathbf{r}_{1} \cdot \mathbf{r}_{2}=\mathbf{r}_{1} \cdot \mathbf{r}_{3}=\mathbf{r}_{2} \cdot \mathbf{r}_{3}=0 \quad \text { and } \quad \mathbf{r}_{1} \cdot \mathbf{r}_{1}-a=\mathbf{r}_{2} \cdot \mathbf{r}_{2}-b=\mathbf{r}_{3} \cdot \mathbf{r}_{3}-c,
$$

where $a \geq b \geq c$ and $a+b+c=0$. Define $M_{\Phi}^{+}$to be the subset of $M_{\Phi}$ where $\mathbf{r}_{1}, \mathbf{r}_{2}, \mathbf{r}_{3}$ form a positively oriented basis of $\mathfrak{g}^{*}$, and $M_{\Phi}^{-}$to be the subset where they form a negatively oriented basis.

It turns out that the hyperkähler structure is nonsingular on $M_{\Phi}^{ \pm}$but singular on the hypersurface dividing them, and the hyperkähler structures on $M_{\Phi}^{ \pm}$have opposite orientations. There are three interesting cases:

Case (A): $a=b=c=0$. In this case, $M_{\Phi}^{ \pm}$are both copies of $\mathbb{H} /\{ \pm 1\}$ with the flat hyperkähler structure, meeting at the origin.

Case (B): $a>0, b=c=-\frac{1}{2} a$. Here $M_{\Phi}^{ \pm}$are copies of the Eguchi-Hanson space [5], which intersect at a common $\mathcal{S}^{2}$ where $\mathbf{r}_{2}=\mathbf{r}_{3}=0$. 
Case (C): $a \geq b>c$. Here $M_{\Phi}$ carries one of the metrics found by Belinskii et al. [1], which has a curvature singularity on the hypersurface $\mathbf{r}_{3}=0$. Note that $M_{\Phi}$ is nonsingular as a submanifold at this hypersurface, even though the hyperkähler structure is singular.

In principle we could follow the construction through to find an explicit algebraic formula for the metrics and complex structures. In the same way, given a semisimple Lie group $G$, one can use this method to construct and classify all hyperkähler manifolds $M$ with dimension $2 \operatorname{dim} \mathfrak{g}-2$ rank $\mathfrak{g}$ and a $G$-action $\rho$ satisfying Condition 12.1.

Now Kronheimer [10], [11], Biquard [3] and Kovalev [9] have already constructed families of hyperkähler manifolds associated to Lie groups, from a completely different point of view. Let $G$ be a compact Lie group with Lie algebra $\mathfrak{g}$, and let the complexification of $G$ be $G^{c}$ with Lie algebra $\mathfrak{g}^{c}$. Kronheimer found that certain moduli spaces of singular $G$-instantons on $\mathbb{R}^{4}$ are hyperkähler manifolds. These moduli spaces can be identified with coadjoint orbits of $G^{c}$ in $\left(\mathfrak{g}^{c}\right)^{*}$, and have hyperkähler metrics invariant under $G$. Kronheimer's construction worked only for certain special coadjoint orbits, and more general cases were handled by Biquard and Kovalev.

Although Kronheimer's metrics look very algebraic, their construction is in fact analytic, and the algebraic description of these metrics is not well understood. All the coadjoint orbit metrics found by Kronheimer, Biquard and Kovalev satisfy Condition 12.1. The manifolds found by Kronheimer [10], [11] have dimension $2 \operatorname{dim} \mathfrak{g}-2$ rank $\mathfrak{g}$, and so Corollary 12.6 applies to give an algebraic construction for Kronheimer's metrics. However, many of the examples of Kovalev and Biquard satisfy $\operatorname{dim} M<2 \operatorname{dim} \mathfrak{g}-2 \operatorname{rank} \mathfrak{g}$, so Corollary 12.6 does not apply. They can be treated algebraically, but in a more complicated way.

In the case $G=S O(3)$ of Example 12.7, case (A) gives the nilpotent orbit metric defined in [11], and case (B) gives the metrics on $G^{c} / T^{c}$ defined in [10], where $T$ is a maximal torus in $G$. However, the metrics of case $(\mathrm{C})$ are not constructed by Kronheimer, Biquard or Kovalev. Thus, in general we expect that most of the metrics constructed by these algebraic methods will be new.

\section{References}

[1] V.A. Belinskii, G.W. Gibbons, D.N. Page, C.N. Pope, 'Asymptotically euclidean Bianchi IX metrics in quantum gravity', Phys. Lett. 76B (1978), 433-435.

[2] K.H. Bhaskara and K. Viswanath, Poisson algebras and Poisson manifolds, Pitman Res. Notes in Math. 174, Longman, 1988.

[3] O. Biquard, 'Sur les équations de Nahm et la structure de Poisson des algèbres de Lie semi-simples complexes', Math. Ann. 304 (1996), 253-276.

[4] C.A. Deavours, 'The quaternion calculus', Amer. Math. Monthly 80 (1973), 995-1008.

[5] T. Eguchi and A.J. Hanson, 'Asymptotically flat solutions to Euclidean gravity', Phys. Lett. 74B (1978), 249-251.

[6] N.J. Hitchin, A. Karlhede, U. Lindström and M. Roček, 'Hyperkähler metrics and supersymmetry', Commun. Math. Phys. 108 (1987), 535-589. 
[7] D.D. Joyce, 'The hypercomplex quotient and the quaternionic quotient', Math. Ann. 290 (1991), 323-340.

[8] D.D. Joyce, 'Compact hypercomplex and quaternionic manifolds', J. Diff. Geom. 35 (1992), 743-61.

[9] A. Kovalev, 'Nahm's equations and complex adjoint orbits', Quart. J. Math. Oxford, 47 (1996), 41-58.

[10] P.B. Kronheimer, 'A hyper-kählerian structure on coadjoint orbits of a semisimple complex group', J. London Math. Soc. 42 (1990), 193-208.

[11] P.B. Kronheimer, 'Instantons and the geometry of the nilpotent variety', J. Diff. Geom. 32 (1990), 473-490.

[12] D. Quillen, 'Quaternionic algebra and sheaves on the Riemann sphere', preprint, 1996.

[13] S.M. Salamon, Riemannian geometry and holonomy groups, Pitman Res. Notes in Math. 201, Longman, 1989.

[14] A. Sudbery, 'Quaternionic analysis', Math. Proc. Camb. Phil. Soc. 85 (1979), 199-225. Lincoln College, Oxford. 